\title{
Chapter 12 \\ Models of the Main Geomagnetic Field Based on Multi-satellite Magnetic Data and Gradients-Techniques and Latest Results from the Swarm Mission
}

\author{
Christopher C. Finlay
}

\begin{abstract}
Magnetic field observations from low-Earth-orbiting satellites provide a unique means of studying ionospheric current systems on a global scale. Such studies require that estimates of other sources of the Earth's magnetic field, in particular, the dominant main field generated primarily in Earth's core but also due to the magnetized lithosphere and large-scale magnetospheric currents, are first removed. Since 1999 multiple low-Earth-orbit satellites including Ørsted, CHAMP, SAC-C, and most recently the Swarm trio have surveyed the near-Earth magnetic field in increasing detail. This chapter reviews how models of the main magnetic field are today constructed from multiple satellites, in particular discussing how to take advantage of estimated field gradients, both along-track and across-track. A summary of recent results from the Swarm mission regarding the core and lithospheric field components is given, with the aim of informing users interested in ionospheric applications of the options available for high accuracy data reduction. Limitations of the present generation of main field models are also discussed, and it is pointed out that further progress requires improved treatment of ionospheric sources, in particular at polar latitudes.
\end{abstract}

\subsection{Introduction}

Ionospheric current systems produce magnetic fields that are measured by magnetometers on low-Earth-orbit satellites, together with the magnetic fields produced by a wide range of other natural sources. The largest of these sources is the so-called 'main' magnetic field, generated in Earth's liquid metal outer core through motional induction in a process known as the geodynamo (e.g. Roberts and King 2013). For those interested in precise studies of ionospheric currents it is important to remove a high-resolution estimate of the internally-generated field, capturing as far as possible its small-scale structure and secular time dependence (see e.g. Stolle et al. 2016).

C. C. Finlay $(\bowtie)$

DTU Space, Technical University of Denmark, Lyngby, Denmark

e-mail: cfinlay@space.dtu.dk 
In the context of this book, models of the main magnetic field can, therefore, be considered as important tools needed for studying ionospheric physics. Moreover, some of the data processing and modelling techniques used in main field studies are themselves of interest to ionospheric physicists, since they can easily be adapted to the study of ionospheric processes.

In this chapter, I begin by reviewing how models of the main geomagnetic field are constructed, focusing on recent developments that take advantage of magnetic field data collected by the low-Earth-orbit Swarm satellite constellation. The aim is to provide an easily accessible account of the construction of advanced main field models, so that users can make a well-informed decision about which models may be most suitable for their specific data processing and reduction tasks. Following this, a survey is given of the latest results regarding the structure and time-dependence of the internal geomagnetic field, as derived from data collected by the Swarm mission. The CHAOS series of field models (Olsen et al. 2006, 2009, 2010, 2014; Finlay et al. 2015, 2016) is a regularly updated, high resolution, main field model that covers the past one and half solar cycles. It will serve here as an illustrative example of an advanced field model that may be of interest for ionospheric studies.

The development of high-resolution geomagnetic field models is a community effort, in particular, facilitated by comparisons carried out within the framework of the International Geomagnetic Reference Field (IGRF) (Thébault et al. 2015a). Aside from the CHAOS model, that is the focus of this chapter, high-resolution field models are also available from a number of other groups, for example, the GRIMM series of models (e.g. Lesur et al. 2008, 2010, 2015b), the POMME series of models (e.g. Maus et al. 2005, 2006b) and the Comprehensive Model/Inversion series of models (e.g. Sabaka et al. 2004, 2015, 2018). Interested readers should consult these references for further details on these models. Limitations of all existing main field models, and opportunities to improve them using our expanding knowledge of ionospheric processes are discussed at the end of this chapter.

\subsection{Fundamentals of Main Field Modelling}

\subsubsection{Calibration of Vector Magnetic Field Measurements}

Modern main field geomagnetic reference models are derived primarily from magnetic field observations collected by low-Earth-orbit satellites. In particular, data from the Swarm satellite constellation, supplemented by measurements made on ground at geomagnetic observatories, are now crucial. For studies of the main field it is essential that the measurements, from both ground and satellite, have absolute accuracy - this is in contrast to the study of ionospheric processes, where often only rapid field variations are of interest. For satellite measurements this involves 
careful magnetometer design, strict magnetic cleanliness procedures when constructing the spacecrafts, pre-flight characterization of stray fields (Jørgensen et al. 2008) and in-flight calibration based on comparisons between fluxgate vector magnetometers and absolute scalar magnetometers that independently measure the field intensity (e.g. Olsen et al. 2003; Yin and Lühr 2011). Accurately orientated vector field measurements are also essential, since using scalar field intensity data alone there is a fundamental ambiguity arising from lack of knowledge perpendicular to the field (Backus 1970; Lowes 1975), particularly at the magnetic equator. In magnetic mapping missions, attitude information is today provided by high precision, non-magnetic, star trackers (Jørgensen et al. 2003). For Swarm, after application of models describing thermal fluctuations, attitude information is available at the arc-second level (Herceg et al. 2017).

Inflight calibration of the vector field data, for example in the case of the Swarm satellites, is carried out by minimizing the difference between the scalar magnetic field $F_{A S M}$ measured by an absolute scalar magnetometer and the magnitude of the vector magnetic field $|\mathbf{B}|$ measured by the vector fluxgate magnetometer (VFM) frame. Free parameters that can be adjusted during inflight calibration arise when relating $\mathbf{B}$ to the vector field $\mathbf{B}_{\text {pre-flight }}$ determined using pre-flight determined fluxgate magnetometer calibration parameters, and after correction for the pre-flight determined stray magnetic fields (e.g. Olsen and Kotsiaros 2011), via the relation

$$
\mathbf{B}=\underline{\underline{P}}^{-1} \underline{\underline{S}}^{-1} \mathbf{B}_{\text {pre-flight }}+\mathbf{B}_{\text {offset }}
$$

where $\underline{S}$ is a $3 \times 3$ diagonal scaling matrix, whose elements can be time dependent. $\underline{P}$ is the non-orthogonality matrix that makes small adjustments to the pre-flight

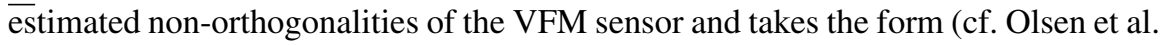
2003)

$$
\underline{\underline{P}}=\left(\begin{array}{ccc}
1 & 0 & 0 \\
-\sin u_{1} & \cos u_{1} & 0 \\
\sin u_{2} & \sin u_{3} & \sqrt{1-\sin ^{2} u_{2}-\sin ^{2} u_{3}}
\end{array}\right)
$$

where $u_{i}$ are parameters describing the rotation to the non-orthogonal magnetic sensor axes coordinate system. $\mathbf{B}_{\text {offset }}$ represents additional small vector offsets/biases. In the case of the Swarm satellites, a small solar-driven magnetic disturbance, thought to be due to currents flowing in the satellite body as a result of thermo-electric effects, was detected post-launch (Tøffner-Clausen et al. 2016). This has been successfully described using an empirical model that depends on the sun position relative to the satellite and it is applied as an additional offset factor during the Level $1 \mathrm{~b}$ magnetic data calibration procedure (for more details see Tøffner-Clausen et al. 2016). Physics-based models of this disturbance have also been developed, and improvements in Swarm's on-board calibration are ongoing. 


\subsubsection{Selection of Magnetic Field Data for Main Field Modelling}

When constructing models of the main field, typically only data from geomagnetically quiet times are used, in an effort to reduce as far as possible the contaminating signatures arising from magnetospheric and ionospheric current systems. Of course this is the opposite mode of operation to that of the space physicist, who is often more interested in data collected during strongly disturbed conditions. Typical quiettime data selection criteria are that the $K_{p}$ index is less than $2 o$, that the rate of change of $D_{s t}$ or similar ring current indices is less than $2 \mathrm{nT} / \mathrm{h}$, and that for data in the polar region that the merging electric field, as determined from solar wind and IMF conditions measured at the $\mathrm{L} 1$ point, is less than $0.8 \mathrm{mV} / \mathrm{m}$ [Olsen et al. (2006), Olsen et al. (2014)]; for a more detailed discussion of data selection in internal field modelling interested readers should consult the recent reviews by Finlay et al. (2017) and Kauristie et al. (2017).

\subsubsection{Potential Field Modelling}

The majority of the main field models presently in operational use assume that the region of interest (where magnetic measurements are collected and where an estimate of the magnetic field is to be made) is current-free, i.e. the current density $\mathbf{J}=0$. Under these conditions, the curl of the vector magnetic field $\mathbf{B}$ is zero

$$
\mu_{0} \mathbf{J}=\nabla \times \mathbf{B}=0
$$

Note that strictly $\mathbf{B}$ should be referred to as the magnetic induction, but in geomagnetism it is for simplicity called the magnetic field. In such source-free regions, it follows that the magnetic field vector $\mathbf{B}$ can be represented as the gradient of a scalar magnetic potential $V$

$$
\mathbf{B}=-\nabla V
$$

where the - sign is included as a matter of convention. Since the magnetic field is also divergence-free

$$
\nabla \cdot \mathbf{B}=0
$$

the scalar potential $V$ must be a solution of Laplace's equation

$$
\nabla \cdot(\nabla V)=\nabla^{2} V=0
$$

In spherical geometry that is relevant for describing the Earth, Laplace's equation takes the form 


$$
\nabla^{2} V=\frac{1}{r^{2}} \frac{\partial}{\partial r}\left(r^{2} \frac{\partial V}{\partial r}\right)+\frac{1}{r^{2} \sin \vartheta} \frac{\partial}{\partial \vartheta}\left(\sin \vartheta \frac{\partial V}{\partial \vartheta}\right)+\frac{1}{r^{2} \sin ^{2} \vartheta} \frac{\partial^{2} V}{\partial \lambda^{2}}=0
$$

where $r$ is the distance from the centre of the Earth, $\vartheta$ is the co-latitude measured from the north pole, and $\lambda$ is the longitude, all defined in an Earth-centred, Earth-fixed, geographic reference frame.

Laplace's equation in spherical geometry (12.6) may be solved by parts (see e.g. Riley et al. 2006); two solutions with different radial dependence are possible, $V^{\text {int }}$, describing internal sources (e.g. due to currents originating in the core or due to the magnetized lithosphere), and $V^{\text {ext }}$, describing external sources (e.g. due to magnetospheric currents)

$$
V=V^{\text {int }}+V^{\text {ext }}
$$

\subsubsection{Representation of the Field Due to Internal Sources}

The solution for the potential due to internal sources takes the form

$$
V^{\mathrm{int}}(r, \vartheta, \lambda, t)=a \sum_{n=1}^{N_{\text {int }}} \sum_{m=0}^{n}\left[g_{n}^{m}(t) \cos m \lambda+h_{n}^{m}(t) \sin m \lambda\right]\left(\frac{a}{r}\right)^{n+1} P_{n}^{m}(\cos \vartheta)
$$

where $a=6371.2 \mathrm{~km}$ is a reference radius, taken to be Earth's mean spherical radius, $(r, \vartheta, \lambda)$ are the geographic coordinates, $P_{n}^{m}$ are Schmidt semi-normalized associated Legendre functions (e.g. Langel 1987; Winch et al. 2005) of degree $n$ and order $m$, and $\left\{g_{n}^{m}, h_{n}^{m}\right\}$ are the Gauss coefficients describing the amplitude of the internal sources. In principle, the maximum degree $N_{\text {int }}$ would be infinity if one wished to represent all possible details of the field structure, but for practical reasons the expansion is truncated at some finite maximum degree, beyond which the smallest wavelengths cannot be reliably retrieved. Illustrative examples of spherical harmonic functions $Y_{n}^{m}(\vartheta, \lambda)$, i.e. $\cos m \lambda P_{n}^{m}(\cos \vartheta)$ or $\sin m \lambda P_{n}^{m}(\cos \vartheta)$ are presented in Fig. 12.1. These are fundamental building blocks that may be combined to represent global functions on a spherical surface.

In addition to spatial dependence, accurate models of the main field must take into account the slow temporal or secular variation of the internal field. In standard models such as the International Geomagnetic Reference Field (IGRF), this is accounted for by linear interpolation between Gauss coefficients $g_{n}^{m}$ defined at reference epochs.

In more advanced models, the time dependence is often represented using B-spline basis functions of order $K$ (de Boor 1978; Bloxham and Jackson 1992) such that

$$
g_{n}^{m}(t)=\sum_{p} g_{n}^{m, p} \mathscr{B}_{K, p}(t)
$$



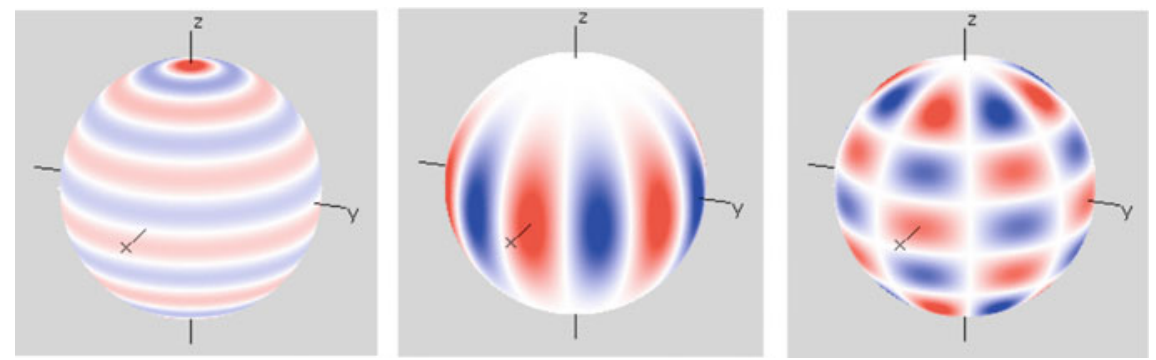

Fig. 12.1 Examples of spherical harmonic basis functions used to parameterize spatial structure in main field models. (Left), a zonal harmonic $n=11, m=0$ (centre) a sectorial harmonic $n=6$, $n=6$, (right), a general tesseral harmonic $n=8, m=4$

Fig. 12.2 Example of 10 cubic B-spline local basis functions that when combined with different weights can be used to represent the

time-dependence of Gauss coefficients in internal field models. For further details, see Jackson and Finlay (2007)

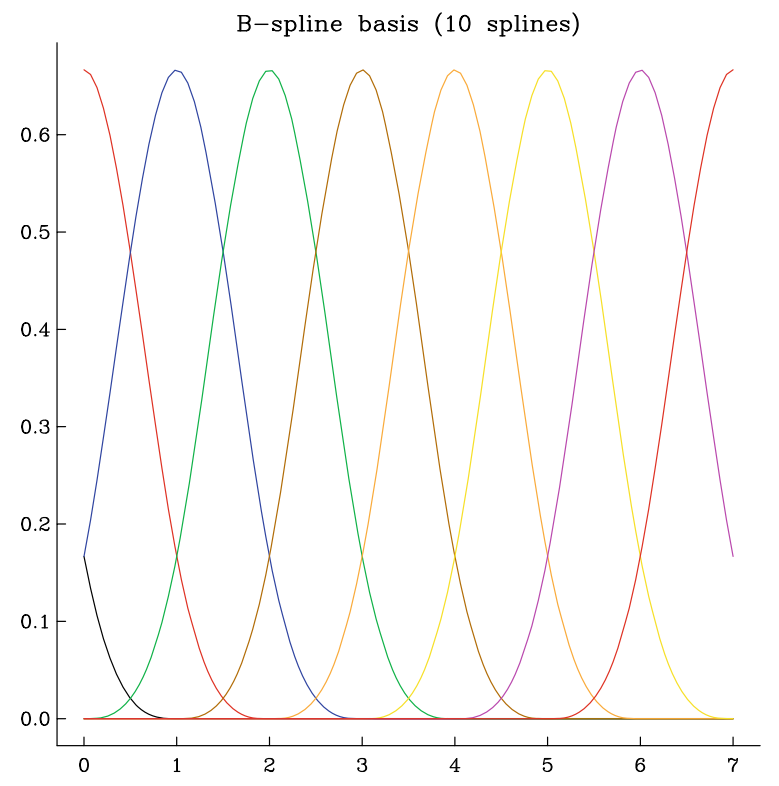

where $g_{n, p}^{m}$ are a set of spline coefficients for each Gauss coefficient $g_{n}^{m}$, defined at knots $p$ that span the time interval of interest. The B-spline basis functions $\mathscr{B}_{K, p}$ are piecewise polynomials of order $K$. Examples of B-spline basis functions of order $K=4$ (i.e. cubic B-splines), that when combined with appropriate weights can reproduce the time-dependent signal of interest, are shown in Fig. 12.2. In advanced main models including the latest members of the CHAOS model series (Olsen et al. 2014; Finlay et al. 2016), order 6 B-splines are used, so that the resulting models can easily be differentiated twice in time to study the field acceleration. 


\subsubsection{Representation of the Field Due to External Sources}

The basic parameterization of magnetic fields due to sources located external to observation points, within the potential field framework, takes the form

$$
V^{\mathrm{ext}}(r, \vartheta, \lambda, t)=a \sum_{n=1}^{N_{\mathrm{ext}}} \sum_{m=0}^{n}\left[q_{n}^{m}(t) \cos m \lambda+s_{n}^{m}(t) \sin m \lambda\right]\left(\frac{r}{a}\right)^{n} P_{n}^{m}(\cos \vartheta)
$$

Although mathematically sufficient, this representation does not account for the specific spatial structure and time dependence of the various magnetospheric current systems.

Maus and Lühr (2005) and Olsen et al. (2005) developed more useful parameterizations of the near-Earth external field including (i) a component expressed in the Solar Magnetic (SM) coordinate system (with its $z$ axis parallel to the Earth's magnetic dipole axis and its $y$ axis perpendicular to the plane containing the dipole axis and the Earth-Sun line) that represents well the geometry of the magnetospheric ring current, and (ii) a part in the Geocentric Solar Magnetospheric (GSM) coordinate system (with its $x$ axis towards the Sun and its $z$ axis being the projection of the Earth's magnetic dipole axis (positive North) on to the plane perpendicular to the $x$ axis) that is more suitable for studying magnetospheric phenomena strongly influenced by the interplanetary magnetic field direction including magnetotail and magnetopause currents. Further details of these and other magnetic coordinate systems, including how to convert between them, are described by Laundal and Richmond (2017).

Taking as a specific example the parameterization of the external field in the latest version of the CHAOS model series (Olsen et al. 2014; Finlay et al. 2016), one may write the external potential as

$$
\begin{aligned}
V^{\mathrm{ext}}(r, \vartheta, \lambda, t)= & a \sum_{n=1}^{N_{S M}} \sum_{m=0}^{n}\left[q_{n}^{m} \cos m T_{d}(t)+s_{n}^{m} \sin m T_{d}(t)\right]\left(\frac{r}{a}\right)^{n} P_{n}^{m}\left(\cos \vartheta_{d}(t)\right) \\
& +a \sum_{n=1}^{N_{G S M}} q_{n}^{0, \mathrm{GSM}} R_{n}^{0}(r, \vartheta, \lambda)
\end{aligned}
$$

where $\vartheta_{d}(t)$ and $T_{d}(t)$ are dipole co-latitude and dipole longitude, respectively, of the $(S M)$ coordinate system. The upper line is the SM dependent part; it is truncated at degree $N_{S M}=2$, and includes a special treatment of the $n=1$ terms (see below). The part in GSM coordinates on the lower line is truncated at degree $N_{G S M}=2$, but is restricted to order $m=0)$. Here the functions $R_{n}^{0}(r, \vartheta, \lambda)$ are modifications of the Legendre functions that explicitly account for field contributions induced in the electrically conducting mantle due to the wobble of the GSM z-axis with respect to the Earth's rotation axis. For a non-conducting Earth, these functions would be $R_{n}^{0}=(r / a)^{n} P_{n}^{0}\left(\cos \vartheta_{G S M}\right)$ where $\vartheta_{G S M}$ is co-latitude in the GSM coordinate system; 
considering a plausible $1 \mathrm{D}$ model of mantle conductivity leads to a representation of $R_{n}^{0}$ similar to the expansion described by Maus and Lühr (2005).

In addition to the time dependence arising for the time variation of $S M$ coordinates in an Earth-fixed, Earth-centred frame (which also results in an induced counterpart), the CHAOS model allows for additional time-dependence of the degree one field in SM coordinates, of the form

$$
\begin{aligned}
& q_{1}^{0}(t)=\hat{q}_{1}^{0}\left[\varepsilon(t)+\iota(t)\left(\frac{a}{r}\right)^{3}\right]+\Delta q_{1}^{0}(t) \\
& q_{1}^{1}(t)=\hat{q}_{1}^{1}\left[\varepsilon(t)+\iota(t)\left(\frac{a}{r}\right)^{3}\right]+\Delta q_{1}^{1}(t) \\
& s_{1}^{1}(t)=\hat{s}_{1}^{1}\left[\varepsilon(t)+\iota(t)\left(\frac{a}{r}\right)^{3}\right]+\Delta s_{1}^{1}(t)
\end{aligned}
$$

where the terms in brackets describe field contributions due to the magnetospheric ring-current and their Earth-induced counterparts as given by the RC index (Olsen et al. 2014). RC is a ground-based index similar to $D_{s t}$ but with better baseline control and including a separation into internal and external parts $R C(t)=\varepsilon(t)+\iota(t)$ based on an a priori model of mantle conductivity. If $R C$ were a perfect description of the magnetospheric field at satellite altitude then the values of the regression coefficients would be $\hat{q}_{1}^{0}=-1, \hat{q}_{1}^{1}=\hat{s}_{1}^{1}=0$ and the 'RC baseline corrections' $\Delta q_{1}^{0}, \Delta q_{1}^{1}$ and $\Delta s_{1}^{0}$ would vanish. The most recent version of the CHAOS model (Finlay et al. 2016) estimated such baseline corrections in bins of 5 days (for $\Delta q_{1}^{0}$ ) and 30 days (for $\Delta q_{1}^{1}, \Delta s_{1}^{1}$ ).

\subsubsection{Using Data in the Magnetometer Frame: Co-estimation of Magnetometer Attitude}

An important issue when using satellite magnetic field data is how to relate the measured field components, made in the reference frame of the magnetometer, $\mathbf{B}_{\mathrm{VFM}}$, to the field components in the Earth-centred, Earth-fixed, North-East-Centre (NEC) frame predicted by global field models $\mathbf{B}_{N E C}=\left(-B_{\vartheta}, B_{\lambda},-B_{r}\right)$. The two representations are related through a rotation that may be expressed in the form

$$
\mathbf{B}_{\mathrm{VFM}}=\underline{\underline{\mathbf{R}}}_{1} \cdot \underline{\underline{\mathbf{R}}}_{2} \cdot \underline{\underline{\mathbf{R}}}_{3} \cdot \mathbf{B}_{\mathrm{NEC}}
$$

Here, the rotation matrix $\underline{\underline{\mathbf{R}}}_{3}$ rotates the magnetic field from the NEC system to the International Celestial Reference Frame (ICRF) and is derived from the satellite position and time (Seeber 2003), $\underline{\underline{\mathbf{R}}}_{2}$ rotates the magnetic field from the (ICRF) system to the Common Reference Frame $(C R F)$ of the satellite's star tracker and is constructed from the attitude data collected by the star tracker, and finally $\underline{\mathbf{R}}_{1}$ rotates from the star tracker $C R F$ to the orthogonal magnetometer (VFM) frame. $\underline{\underline{\mathbf{R}}}_{3}$ and 
$\underline{\underline{\mathbf{R}}}_{2}$ are typically well known and in many cases it is also assumed that $\underline{\underline{\mathbf{R}}}_{1}$ is known exactly and in advance of collecting the data. However, in the most advanced main field models including the CHAOS series, rather than being assumed in advance, the Euler angles defining $\underline{\underline{\mathbf{R}}}_{1}$ are instead co-estimated as part of the modelling procedure (Olsen et al. 2006). In this case, the relation between the measurements in the VFM frame and the model parameters (coefficients of the internal potential from (12.1) and (12.9), of the external potential from (12.11) and (12.12), and the Euler angles defining $\underline{\underline{\mathbf{R}}}_{3}$ from (12.13), is nonlinear, and model estimation becomes a nonlinear inverse problem.

\subsubsection{Model Estimation: Solution of the Inverse Problem}

The relationship between the predicted vector magnetic field data in the vector fluxgate magnetometer (VFM) frame, listed as a vector $\mathbf{d}_{\text {mod }}$ and the model parameters m may compactly be written in the form

$$
\mathbf{d}_{\text {mod }}=\mathbf{g}(\mathbf{m})
$$

where $\mathbf{g}(\mathbf{m})$ denotes the non-linear dependence on the model parameters $\mathbf{m}$.

Determination of the model parameters from the magnetic observations $\mathbf{d}_{\text {obs }}$ is a non-linear inverse problem. Furthermore, since it involves downward continuation of observations from satellite altitude, it is also an ill-conditioned problem. Moreover, since there are field sources that vary rapidly in space and time that cannot be captured by the model (i.e. the model is incomplete, for example, failing to account for auroral and polar-cap currents) the residuals between model predictions and the data are often long-tailed and not simply Gaussian distributed.

A well-proven technique for finding suitable solutions to this difficult estimation problem is the regularized, robust, iteratively reweighted least squares (IRLS) algorithm. This approach, which is adopted in the construction of the CHAOS field model series, involves iteratively minimizing a cost function of the form

$$
\mathscr{J}=\mathbf{e}^{T} \underline{\underline{\underline{W}}} \mathbf{e}+\lambda_{3} \mathbf{m}^{T} \underline{\underline{\Lambda}}_{3} \mathbf{m}+\lambda_{2} \mathbf{m}^{T} \underline{\underline{\Lambda}}_{2} \mathbf{m},
$$

where $\mathbf{e}=\mathbf{d}_{\text {obs }}-\mathbf{d}_{\text {mod }}=\mathbf{d}_{\text {obs }}-\mathbf{g}(\mathbf{m})$ is the vector of residuals (observations minus the model predictions). $\underline{\underline{\mathbf{W}}}=\underline{\underline{\mathbf{C}}}^{-1 / 2} \underline{\underline{\mathbf{H}}} \underline{\underline{\mathbf{C}}}^{-1 / 2}$ is a data weighting matrix, derived from a pre-specified data error covariance matrix $\underline{\underline{\mathbf{C}}}$ and $\underline{\underline{\mathbf{H}}}$, a diagonal matrix of residualdependent weights, for example, $H_{j j}=\min \left(1, c_{H} \sigma_{j} /\left|e_{j}^{i}\right|\right)$ with $c_{H}=1.5, e_{j}^{i}$ the residual of the $j$ th data at the previous (i.e. ith) iteration (e.g. Constable 1988; Olsen 2002) and $\sigma_{j}$ is an a priori estimate of data uncertainty for the $j$ th datum. $H_{j j}$ are known as Huber weights, and they permit robust solutions to be obtained even in the presence of long-tailed error distributions. 
$\mathbf{m}^{T} \underline{\underline{\Lambda}}_{3} \mathbf{m}$ is a quadratic norm measuring the mean squared magnitude of the third time derivative of the radial field $\left|\frac{\partial^{3} B_{r}}{\partial t^{3}}\right|$, integrated over the surface of the outer core $\Omega_{c}$ (radius $c=3485 \mathrm{~km}$ ) and time-averaged over the time span of the model between $t_{\text {start }}$ and $t_{\text {end }}$

$$
\left\langle\left|\frac{\partial^{3} B_{r}}{\partial t^{3}}\right|^{2}\right\rangle=\frac{1}{\left(t_{\text {start }}-t_{\text {end }}\right)} \int_{t_{\text {start }}}^{t_{\text {end }}} \int\left|\frac{\partial^{3} B_{r}}{\partial t^{3}}\right|^{2} d \Omega_{c} d t=\mathbf{m}^{T} \underline{\underline{\Lambda}}_{3} \mathbf{m}
$$

Finally, $\mathbf{m}^{T} \underline{\Lambda}_{2} \mathbf{m}$ is a similar measure of the mean square magnitude of the second time derivative or secular acceleration of the radial field at the core surface $\left|\frac{\partial^{2} B_{r}}{\partial t^{2}}\right|$, but evaluated only at the model endpoints $t_{\text {start }}$ and $t_{\text {end }} . \lambda_{3}$ and $\lambda_{2}$ are regularization parameters for these two norms, specifying their relative importance and chosen to ensure a good balance between fitting field time changes (for example, as seen at ground observatories) while at the same time ensuring that spurious temporal variations of the model are suppressed.

Since (12.14) is a (weakly) nonlinear function of the model parameters, an iterative approach is adopted to linearize it, about the present model $\mathbf{m}_{i}$. The model is iteratively updated until convergence of the model parameters (e.g. Gubbins 2004), such that

$$
\mathbf{m}_{i+1}=\mathbf{m}_{i}+\delta \mathbf{m}_{i}
$$

where

$$
\begin{gathered}
\delta \mathbf{m}_{i}=\left[\underline{\underline{\mathbf{G}}}_{i}^{T} \underline{\underline{\mathbf{W}}} \underline{\underline{\mathbf{G}}}_{i}+\lambda_{3} \underline{\underline{\Lambda}}_{3}+\lambda_{2} \underline{\underline{\Lambda}}_{2}\right]^{-1}\left[\underline{\underline{\mathbf{G}}} i \underline{\underline{\mathbf{W}}}\left(\mathbf{d}_{\mathrm{obs}}-\mathbf{g}\left(\mathbf{m}_{i}\right)\right)-\lambda_{3} \underline{\underline{\Lambda}}_{3} \mathbf{m}_{i}-\lambda_{2} \underline{\underline{\Lambda}}_{2} \mathbf{m}_{i}\right], \\
\text { and } \quad \underline{\underline{\mathbf{G}}}_{i}=\left.\frac{\partial \mathbf{g}(\mathbf{m})}{\partial \mathbf{m}}\right|_{\mathbf{m}=\mathbf{m}_{i}} .
\end{gathered}
$$

Given its importance, it is worth explicitly stating here how elements of $\underline{\underline{G}}_{i}$ are computed. As example, constructing three rows of $\underline{\underline{\mathbf{G}}}_{i}$ associated with three $\underline{\overline{\mathrm{C}}}_{i}$ imponents of a vector magnetic field measurement in the VFM frame involves (i) taking the spherical polar gradients of the expansions for internal and external potentials (12.8) and (12.11), (ii) multiplying these with the Euler rotation matrices $\underline{\underline{\mathbf{R}}}_{1} \cdot \underline{\underline{\mathbf{R}}}_{2} \cdot \underline{\underline{\mathbf{R}}}_{3}$ from the previous iteration to rotate to the VFM frame, then (iii) taking the derivatives with respect to each model parameter in turn, such that each column of $\underline{\underline{\mathbf{G}}}_{i}$ refers to a derivative with respect to a different model parameter), and evaluating $\underline{\overline{t h}}_{i}$ e elements using the model parameters from the previous iteration $\mathbf{m}_{i}$. 


\title{
12.3 Use of Field Gradients and Multi-satellite Data in Main Field Modelling
}

\begin{abstract}
Above we have described the standard approach to geomagnetic field modelling, based on the observed vector or scalar field components. The launch of the Swarm multi-satellite constellation (Friis-Christensen et al. 2006; Olsen et al. 2016b), has opened exciting new possibilities for using approximate field gradients, estimated via along-track and across-track differences, in main field modelling. In this section, we outline these new techniques, focusing on how to construct suitable estimates of the field gradients and on how to deal with data from multiple satellites within a single inversion.
\end{abstract}

\subsubsection{Estimates of Field Gradients: Approximation by Along-Track and Across Track Differences}

The constellation of the Swarm trio of satellites, with two satellites (Alpha and Charlie) flying close together and a third (Bravo) flying at higher altitude and drifting in local time with respect to the lower pair, enables estimates both along-track and cross-track gradients of the geomagnetic field to be made from space. In particular, considering differences in the field recorded by the lower pair permits the crosstrack field gradient to be estimated for the first time. This is extremely valuable for constraining small-scale east-west structures in the lithospheric field (Maus et al. 2006a; Thébault et al. 2016), although it provides no constraint on zonal $(m=0)$ and little constraint on near zonal (small $m$ ) spherical harmonic components of the field.

Although the Swarm constellation does not have an along-track satellite pair (which would have an approximately north-south orientation at mid and low latitudes), one can instead use along-track differences from a single satellite to estimate the gradient in this direction, with the assumption the field does not change appreciably over the time taken to move between the locations differenced. A typical time between the locations differenced is $15 \mathrm{~s}$ (Olsen et al. 2015), much shorter than the time scale of large-scale magnetospheric field changes. This corresponds to an along-track spatial separation of about $115 \mathrm{~km}$. (Kotsiaros et al. 2014) have explored the impact on lithospheric field models of using different time separation when computing along-track gradient estimates. There is clearly a trade-off between the signal amplitude (smaller for shorter time differences) and noise (larger for larger timedifferences due to the breakdown of the stationarity assumption). The optimal time separation will also depend on the target wavelengths, on the geomagnetic latitude and on geomagnetic conditions (quiet or storm-time, dark or sunlit). There is certainly room to better optimize the calculation of field gradient estimates. Nonetheless, use of field gradients has already proven to be of great value in deriving high-resolution 
models of the core field (Olsen et al. 2015; Finlay et al. 2016) and lithospheric field (Sabaka et al. 2015; Kotsiaros 2016; Olsen et al. 2017).

How can one use field gradient estimates in the construction of main field models? The approach is a straightforward extension of standard procedure of performing least-square fits. One simply minimizes the square of the residuals between the observed and modelled field differences, which may be either differences of vector components or differences of scalar intensity values.

If we represent residuals between the observed and modelled field differences by

$$
\Delta \mathbf{e}=\Delta \mathbf{d}-\Delta \mathbf{d}_{\bmod }
$$

where $\Delta \mathbf{d}=\mathbf{d}\left(\mathbf{r}_{1}, t_{1}\right)-\mathbf{d}\left(\mathbf{r}_{2}, t_{2}\right)$ and $\Delta \mathbf{d}_{\text {mod }}=\mathbf{d}_{\text {mod }}\left(\mathbf{r}_{1}, t_{1}\right)-\mathbf{d}_{\text {mod }}\left(\mathbf{r}_{2}, t_{2}\right)$ where $\left(\mathbf{r}_{1}, t_{1}\right)$ is the location and time of the first datum contributing to the field difference while $\left(\mathbf{r}_{2}, t_{2}\right)$ is the location and time of the second datum that is subtracted from the first. Minimizing the sum of the squares of the field difference residuals $\Delta \mathbf{e}$ then results in an additional contribution to the cost function (12.14) from fitting field differences that may be written

$$
\Delta \mathbf{e}^{T} \underline{\underline{W_{\Delta}}} \Delta \mathbf{e}
$$

where

$$
\Delta \mathbf{d}_{\text {mod }}=\mathbf{g}_{\Delta}(\mathbf{m}) \quad \text { with } \quad \mathbf{g}_{\Delta}(\mathbf{m})=\left.\mathbf{g}\right|_{\left(\mathbf{r}_{1}, t_{1}\right)}(\mathbf{m})-\left.\mathbf{g}\right|_{\left(\mathbf{r}_{2}, t_{2}\right)}(\mathbf{m})
$$

and similar to previously ${\underline{\underline{W_{\Delta}}}}={\underline{\underline{C_{\Delta}}}}^{-1 / 2} \underline{\underline{H}}_{\Delta}{\underline{\underline{C_{\Delta}}}}^{-1 / 2}$ is a data weighting matrix, derived from a specified data error covariance matrix for the difference data $\underline{\underline{C_{\Delta}}}$ and $H_{\Delta}$, a diagonal matrix of 'Huber' weights $H_{j j}=\min \left(1, c\left(\sigma_{\Delta}\right)_{j} /\left(\Delta e_{j}^{i}\right)\right)$ for the difference data that depends on the amplitude of the residuals at the previous iteration $\Delta e_{j}^{i},\left(\sigma_{\Delta}\right)_{j}$ is the a priori difference data uncertainty estimate and $c_{H}$ is a constant, usually set to 1.5 .

In the field models CHAOS-6 (Finlay et al. 2016), SIFM (Olsen et al. 2015) and SIFM+ (Olsen et al. 2016a), along-track (or approximately north-south at the equator so denoted north-south below) gradients were approximated by the differences $\Delta B_{\mathrm{NS}}= \pm\left[B_{k}\left(t_{k}, r_{k}, \theta_{k}, \phi_{k}\right)-B_{k}\left(t_{k}+15 \mathrm{~s}, r_{k}+\delta r, \theta_{k}+\delta \theta, \phi_{k}+\delta \phi\right)\right]$ of subsequent data, measured by the same satellite $k, 15 \mathrm{~s}$ later, corresponding to an alongtrack distance of $\approx 115 \mathrm{~km}\left(\approx 1^{\circ}\right.$ in latitude near the equator) for the Swarm satellites. Here $B$ could be the scalar intensity $F$ or any of the geocentric field components $\left(B_{r}, B_{\theta}, B_{\phi}\right)$. The sign of the difference was chosen positive if $\delta \theta>0$, otherwise negative. The choice of $15 \mathrm{~s}$ was found to give a reasonable amplitude of internal field signal while being sufficiently short that much of the large-scale external field is unchanged, so therefore removed on taking the difference. $15 \mathrm{~s}$ differences have the advantage of involving differences over lengths similar to the East-West spacing between Swarm's lower satellite pair. 
To approximate the East-West gradients the above studies used the difference $\delta B_{\mathrm{EW}}= \pm\left[B_{A}\left(t_{1}, r_{1}, \theta_{1}, \phi_{1}\right)-B_{C}\left(t_{2}, r_{2}, \theta_{2}, \phi_{2}\right)\right]$, between field components, or the scalar field, measured by the two satellites Swarm Alpha and Swarm Charlie. Here $t_{i}, r_{i}, \theta_{i}, \phi_{i}, i=1-2$ are time, radius, geographic co-latitude and longitude of the two observations. The sign of the difference was chosen such that $\delta \phi=\phi_{1}-\phi_{2}>0$. For each observation $B_{A}$ (from Swarm Alpha) the corresponding value $B_{C}$ (from Swarm Charlie) was chosen to be that closest in co-latitude $\theta$, with the requirement that $|\delta t|=\left|t_{1}-t_{2}\right|<50 \mathrm{~s}$. Note there is a time delay between the ground-tracks of Swarm Alpha and Swarm Charlie to avoid collisions at the pole, so simultaneous values from each satellite do not provide an estimate of the east-west gradient. Taking Swarm Charlie values slightly delayed, typically by around $10 \mathrm{~s}$, allows differences to be taken that are very close to the desired East-West configuration. Note that this again requires that large-scale field be stationary over approximately $10 \mathrm{~s}$ if they are to cancel on taking the difference.

Turning to the estimation of the field model, including field differences in the inversion simply requires that we augment the vectors and matrices used for conventional field modelling, so that these also contain the data and associated entries of the design matrices appropriate for vector and scalar field differences. The algorithm for updating the model using this augmented dataset (containing both field values and field differences) may be written as

$$
\begin{gathered}
\delta \mathbf{m}_{i}=\left[\underline{\underline{\mathbf{G}}}_{i}^{\text {aug T}} \underline{\underline{\mathbf{W}}} \underline{\underline{\mathbf{G}}}_{i}^{\text {aug }}+\lambda_{3} \underline{\underline{\Lambda}}_{3}+\lambda_{2} \underline{\underline{\Lambda}}_{2}\right]^{-1}\left[\underline{\underline{\mathbf{G}}}_{i}^{\text {aug } T} \underline{\underline{\mathbf{W}}}\left(\mathbf{d}_{\mathrm{obs}}^{\text {aug }}-\mathbf{d}_{\text {mod, } \mathrm{i}}^{\text {aug }}\right)-\lambda_{3} \underline{\underline{\Lambda}}_{3} \mathbf{m}_{i}-\lambda_{2} \underline{\underline{\Lambda}}_{2} \mathbf{m}_{i}\right] \\
\text { where } \underline{\underline{\mathbf{G}}}_{i}^{\text {aug }}=\left[\begin{array}{c}
\left.\frac{\partial \mathbf{g}(\mathbf{m})}{\partial \mathbf{m}}\right|_{\mathbf{m}=\mathbf{m}_{i}} \\
\left.\frac{\partial \mathbf{g}_{\Delta}(\mathbf{m})}{\partial \mathbf{m}}\right|_{\mathbf{m}=\mathbf{m}_{i}}
\end{array}\right], \mathbf{d}_{\mathrm{obs}}^{\text {aug }}=\left[\begin{array}{c}
\mathbf{d}_{\mathrm{obs}} \\
\Delta \mathbf{d}_{\mathrm{obs}}
\end{array}\right], \text { and } \mathbf{d}_{\text {mod,i }}^{\text {aug }}=\left[\begin{array}{c}
\mathbf{d}_{\text {mod,i }} \\
\Delta \mathbf{d}_{\text {mod, }, \mathrm{i}}
\end{array}\right]
\end{gathered}
$$

\subsubsection{Information Content of Field Gradient Estimates}

Olsen et al. (2017) have explored in detail how much field models can be improved by using along-track (north-south) and across-track (east-west) gradient estimates, considering both CHAMP and Swarm data in the context of studying the lithospheric field. Figure 12.3, reproduced from their study, shows theoretical model variances of spherical harmonic coefficients as a function of degree (y-axis) and order ( $\mathrm{x}$-axis), based on the positions of CHAMP and Swarm data, and considering the impact of both vector data and vector gradients, i.e. north-south and east-west gradients. Blue colours show well determined coefficients, yellow colours indicate poorly determined model coefficients.

The results in Fig. 12.3 are based on a simplified linearized version of the inverse problem, where the Euler angles are assumed known and only the internal potential is considered. The plots show diagonal entries of the formal model covariance matrix $\left(\mathbf{G}^{T} \mathbf{W G}\right)^{-1}$, where $\mathbf{G}$ here is simply the matrix for the linear forward problem 

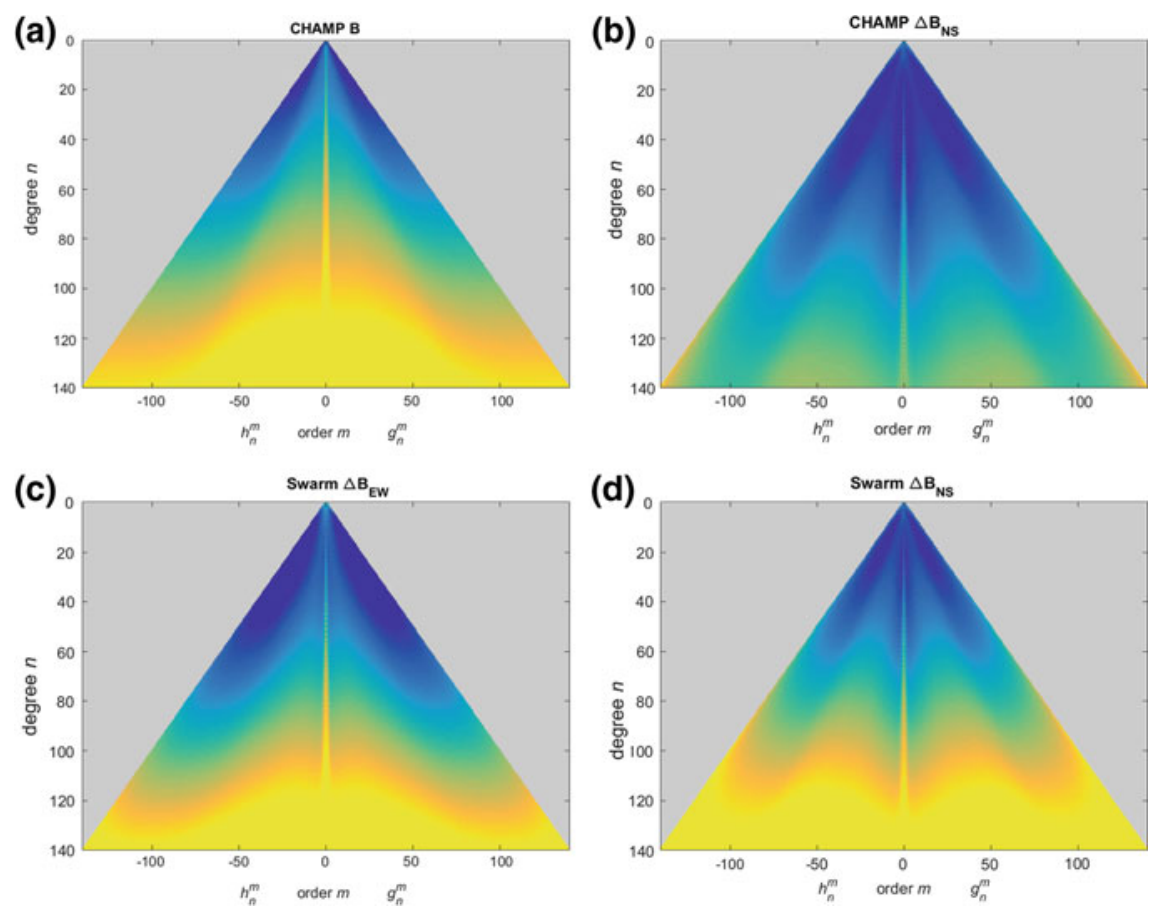

Fig. 12.3 Variance of the spherical harmonic coefficients $g_{n}^{m}, h_{n}^{m}$ derived with various choices of input data sets. Blue corresponds to well determined coefficients, yellow corresponds to poorly determined coefficients. Reproduced from Olsen et al. (2017)

connecting the satellite data (either vector field or vector field differences from either CHAMP and Swarm) to the spherical harmonic model coefficients, and where $\mathbf{W}$ is a matrix of data weights.

North-south or along-track gradients of CHAMP data clearly provide a much improved resolution (i.e. lower model variances) for the high degree spherical harmonic coefficients, especially at degrees 120-140 which are poorly constrained by field data alone. North-south differences do not, however, constrain well the sectorial and near sectorial spherical harmonics. East-West differences from Swarm provide very valuable new constraints on these coefficients. The information provided by Swarm on the high degree field will, of course, be enhanced as the satellites descend to lower altitudes (the greater information provided by CHAMP north-south differences compared to Swarm north-south differences is purely due to the present higher altitude of the Swarm satellites). The poorer determination of high degree near-zonal coefficients is a consequence of the polar gap in the data distribution. Field differences are seen to provide useful information, not just on high degree coefficients associated with the lithospheric field signal, but also on low degree coefficients describing the core field; moreover, unmodelled large-scale magnetospheric fields are effectively 
suppressed when considering field gradient estimates, which increases the signal to noise ratio.

\subsubsection{Examples of Field Gradient Data and Their Interpretation}

In order to illustrate the form of signals seen in field gradient (i.e. north-south and east-west difference) data, and how these are related to field data, we present here scalar field and scalar field difference signals collected by the Swarm satellites (northsouth difference, labelled NS and east-west differences, labelled EW, divided by the separation distance in kilometers between the measurements) on example day-side (Fig. 12.4) and night-side (Fig. 12.5) half orbits. We present the signal remaining after the progressive removal of the estimates of the core field (top), core and lithospheric fields (middle), and core, lithospheric and magnetospheric fields (bottom) as a function of quasi-dipole (QD) latitude. These examples are taken from Olsen and Stolle (2017).

The selected day-side orbit shown in Fig. 12.4 has an equatorial Local Time crossing at 12:12 LT, and is from 2 May 2014, which was a geomagnetically quiet day (Kp $<1+$ and Dst $>-13 \mathrm{nT}$ ). The left column presents observations from Swarm Alpha; the middle column presents estimates of the East-West field difference measured between Swarm Alpha and Swarm Charlie, divided by the distance between the two spacecraft; the right column shows an estimate of the North-South gradient, obtained from 15-second north-south differences of Swarm Alpha divided by d $=141 \mathrm{~km}$ (which is the distance of two satellite measurements taken $15 \mathrm{~s}$ apart). For each of the three columns, the blue curves show the difference $F=F_{\text {obs }}-F_{\text {mod }}$ between the observed magnetic intensity $F_{\text {obs }}$ and various model values $F_{\text {mod }}$ predicted by CHAOS-6 model, whereas the red curves show predictions of some additional parts of the model. The yellow curve in the bottom left panel shows $F_{o b s}$ from Swarm Charlie. See figure caption for more details. For this day-side half orbit, the signature of the equatorial electrojet is clearly seen at the magnetic equator, especially in the north-south gradient (right column), resulting from the depression it causes in the field intensity (see left column). The signature of the Sq ionospheric current system is also seen as minima in field intensity close to $30^{\circ}$ QD latitude. Large signals remain in the polar regions even after removal of estimates of the core, lithospheric, magnetospheric and $\mathrm{Sq}$ fields, especially in sunlit northern hemisphere, where large gradients are seen in both the east-west and north-south differences.

Next, we move to an example night-side half orbit, more typically of the data used in main field modelling. As shown in Fig. 12.5, there is no longer any equatorial electrojet signal at low latitudes or any Sq signal at mid-latitudes. There are, however, some recognizable signals remaining, even after the removal of the core, lithosphere, magnetospheric and Sq-induced signals (note the latter is nonzero, even in dark 

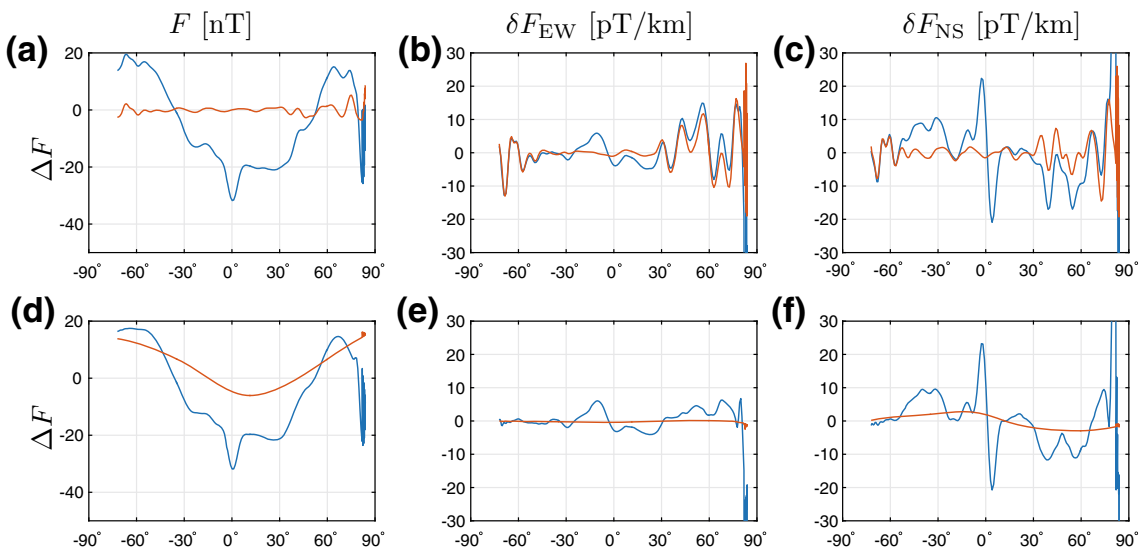

(e)

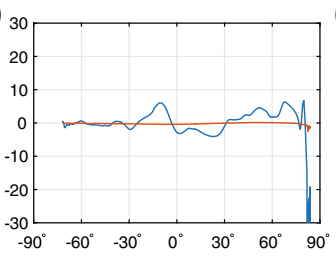

(f)
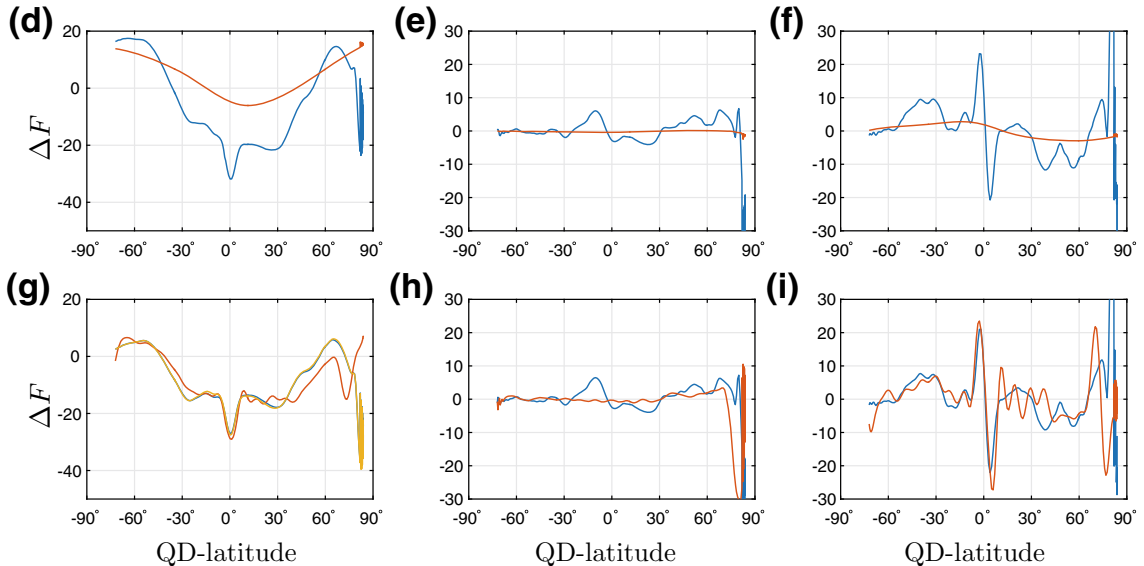

(h)

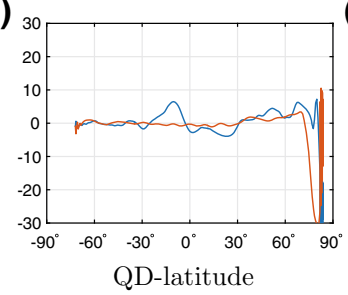

(i)

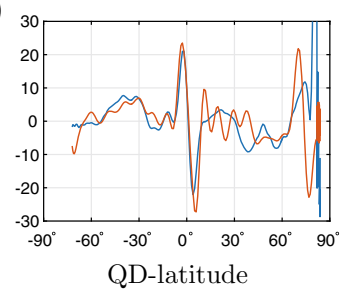

Fig. 12.4 Adapted from Olsen and Stolle (2017). Magnetic field intensity residuals for the daytime part of Swarm orbit number 2464 of 2 May 2014 versus QD latitude. Equator crossing at 18:43:04 UT, corresponding to 12:12 Local Time. a-c: The blue curve shows the difference $\mathrm{F}=$ $F_{o b s}-F_{c o r e}$ between observed magnetic intensity $F_{o b s}$ and the core field part $F_{c o r e}$ as given by the CHAOS-6 model. The red curve shows the lithospheric field model predictions. d-f: The blue curve presents the difference between the two curves of panels $(\mathbf{a}-\mathbf{c})$, i.e. the observed values minus model values for core and lithosphere. The red curve shows the modelled contributions of magnetospheric currents. g-i: The magnetic field intensity after removal of core, lithospheric and magnetospheric model values (shown by the red curves in panels $\mathbf{d}-\mathbf{f}$ ) is shown in blue. The red curves here present ionospheric current contributions as given by the CM5 model. Left panel shows values for Swarm Alpha; middle panel presents East-West gradients based on data from Swarm Charlie minus Swarm Alpha; right panel shows north-south gradients of Swarm Alpha

regions). This is especially evident in the field differences, for example there are characteristic spikes around QD latitude $15^{\circ}$ south in the EW differences that are likely due to ionospheric F-region currents related to steep plasma gradients at postsunset times (these are also obvious in electron density data collected by the Swarm satellites, not shown). Ionisation anomalies after sunset are frequently affected by plasma density irregularities (sometimes called 'bubbles') close to $\pm 10^{\circ}$ QD latitude that produce magnetic signatures of a few nanotesla; these anomalies have small length scale, so can be different between Swarm Alpha and Charlie, that results in clear signatures in the EW differences. Once again the largest unmodelled signals after the removal of the core, lithospheric, magnetospheric and Sq-induced parts, both for field data and field gradient estimates, are found in the polar regions, particularly in 

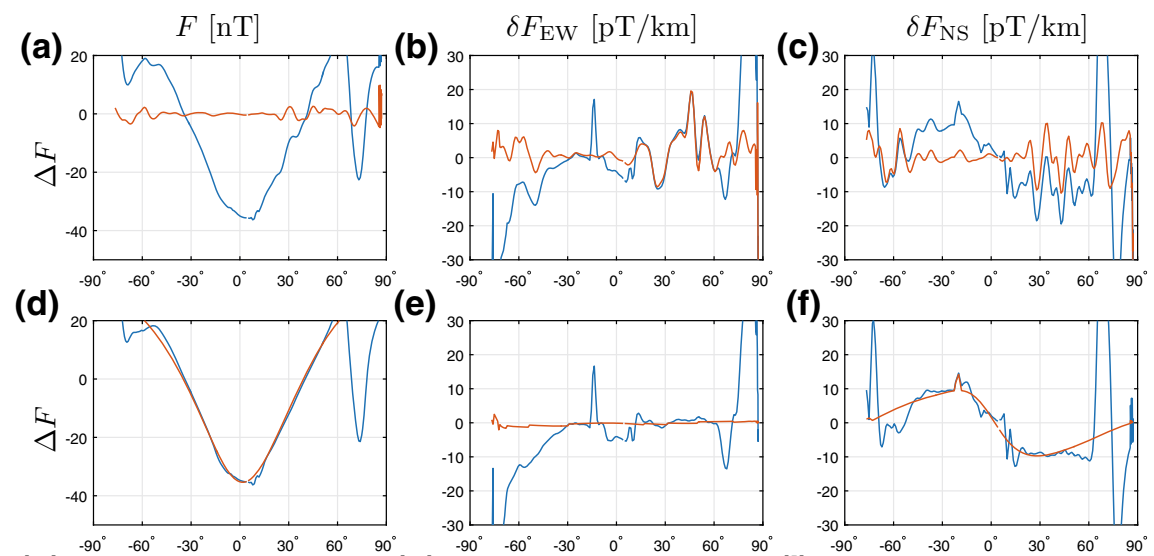

(e)
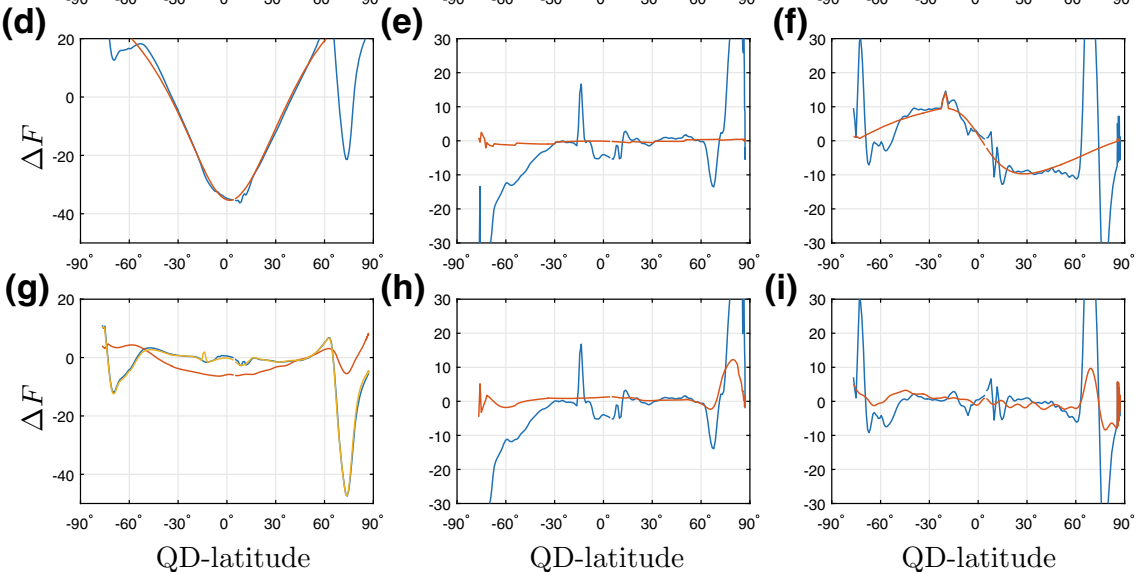

(h)
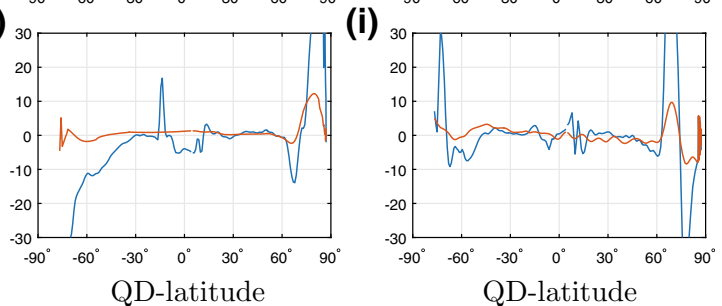

Fig. 12.5 Adapted from Olsen and Stolle (2017). Orbit number 5151 on 25 October 2014. Equator crossing at 01:16:09 UT, corresponding to 20:30 Local Time. Same format as Fig. 12.4

the summer (northern) hemisphere in the example presented here. These unmodelled polar signals are presently the major challenge facing main field modellers (e.g. Finlay et al. 2017).

\subsubsection{Simultaneous Inversion of Data from Multiple Satellites}

Above we have seen the complexity of the signals contained in low-Earth-orbit satellite data, and how the signal from various sources are either suppressed or enhanced when considering field gradient estimates. A crucial step in using all this information, and in combining such information from multiple satellite missions, is defining suitable data error budgets through the weight matrix $\mathbf{W}$. For example, in the recent lithospheric field model LCS-1 (Olsen et al. 2017), an error budget for each component of gradient data for each satellite was developed as a function of quasi-dipole latitude, since many unmodelled near-Earth current systems are organized by the main field geometry. Data uncertainties $\sigma$ (i.e. the square root of the diagonal entries 

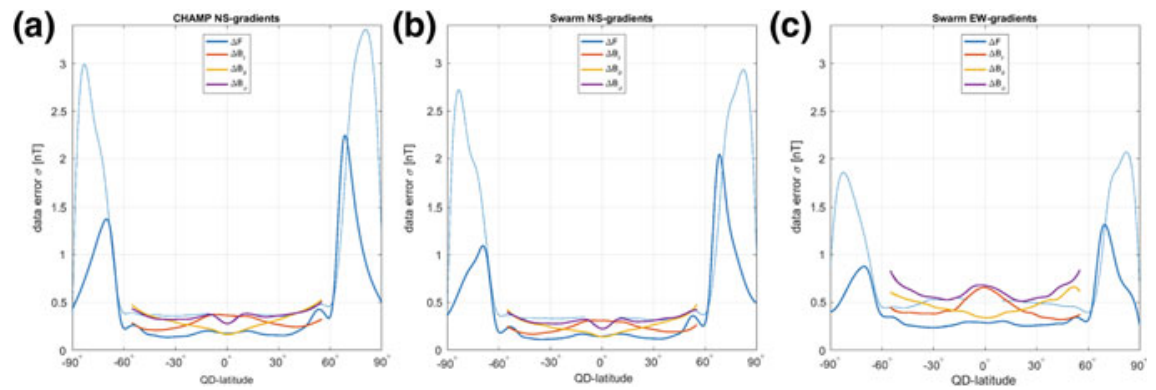

Fig. 12.6 Assigned data uncertainties $\sigma$ for CHAMP(left, north-south gradients), and Swarm (centre, north-south gradients, right, East-West gradients), versus quasi-dipole latitude. The thin blue line represents scalar gradient data under sunlit conditions. Adapted from (Olsen et al. 2017)

in the data covariance matrix $\mathbf{C}$ that along with Huber weights $H_{j j}$ make up W), as employed in the LCS-1 model, are presented in Fig. 12.6.

Separate uncertainty estimates for CHAMP north-south gradients, Swarm northsouth gradients and Swarm east-west gradients are specified. Uncertainties for the scalar field are shown separately for sunlit and dark regions. These uncertainties were derived by binning the data residuals relative to the CHAOS- 6 field model, and estimating the standard deviation $\sigma$ using a robust (Huber weighting) approach within bins of $5^{\circ} \mathrm{QD}$ latitude. The largest uncertainties occur in the polar regions, in particular for the sunlit parts, due to the enhanced ionospheric conductivity in these regions, and related magnetosphere-ionosphere coupling. The estimated uncertainties in Swarm inter-satellite East-West differences are generally larger than the northsouth single satellite differences, and there is also interesting evidence for distinct unmodelled signals in the East-West differences at low quasi-dipole latitudes.

Using these uncertainties within the main or lithospheric field inverse problem essentially downweights data from the polar regions that is more likely to be contaminated by the signature of currents not included in the main or lithospheric field model. Data uncertainties allocated from CHAMP and Swarm turn out to be rather similar, showing similar trends as a functions of quasi-dipole latitude. It is also possible to define data and uncertainties from other satellite missions (Ørsted, SAC-C, DMSP etc.), where the uncertainties can be much larger, particularly with regard to how attitude errors influence our determination of the vector field (Olsen et al. 2006).

The error budgets presented here are still rather crude. In reality data errors with respect to internal field modelling are correlated in both space and time due to the structured nature of the unmodelled magnetospheric and ionospheric currents. In particular, the data are correlated along track (Lowes and Olsen 2004) which is why considering along-track or north-south field differences (which decorrelates this error source) is such an advantage. In addition, measurements from similar quasi- 
dipole latitudes and similar local times are likely to have correlated errors that are not presently taken into account.

\subsection{The Internal Field as Seen by the Swarm Multi-satellite Mission}

Having now set out the techniques used to construct advanced models of the internal field from multi-satellite data and gradient estimates, we now move on to give a brief summary the latest knowledge from such models.

For many interested in the Earth's magnetic field, either at present its present state or its changes over the past century, the IGRF is a well known and reliable source of information. It is an IAGA/IUGG endorsed model, produced by a international group of scientists every five years from candidate models. It describes the main field up to spherical harmonic degree $n=13$ and the linear rate of change of the field for the upcoming five years up to degree $n=8$. The most recent 12 th-generation update of IGRF (Thébault et al. 2015b) used data from CHAMP, Swarm and ground observatories to provide estimates of the field in 2010, 2015 and a predicted field change for 2015-2020. The advantage of IGRF is that it is an internationally agreed reference. However, it fails to describe the small scale lithospheric field, and it does not catch nonlinear secular variation, including geomagnetic jerk events.

For applications in detailed studies of ionospheric current systems, advantages have been documented in reducing data using more advanced field models that include the small-scale lithospheric field, estimates of the large magnetospheric field, and that follow fast changes in the core field (Stolle et al. 2016; Alken 2016). Such advanced field models include the POMME model developed by Maus and co-workers (Maus et al. 2005, 2006b, 2010), the GRIMM model produced by Lesur and co-workers (Lesur et al. 2008, 2010, 2015a) and the CHAOS model produced by Olsen and colleagues (Olsen et al. 2006, 2009, 2010, 2014; Finlay et al. 2015, 2016). The Comprehensive model series, developed by Sabaka and co-workers (Sabaka et al. 2002, 2004, 2013, 2015), takes an alternative approach and seeks to co-estimate not only the internal field but as far as possible all near-Earth field sources, including ionospheric and oceanic tidal sources. For further details on these models and their differences, the interested reader should consult the above references.

Here, we present the current state of knowledge of the core field, as determined from the latest Swarm data in the CHAOS-6 field model (Finlay et al. 2016), and a recent image of the global lithospheric field, from the LCS-1 field model (Olsen et al. 2017), based on the latest data from CHAMP and Swarm. 


\subsubsection{The Core Field}

The main part of the geomagnetic field is generated within the liquid iron core of the Earth. Knowledge of the core dynamo process is obtained by downward continuing the internal part of field models to the outer edge of the source region, at the coremantle boundary (a radius of approx. $3480 \mathrm{~km}$ ). In doing this, it is assumed that there are no current sources in the mantle on the time scales studied. Maps of the radial component of the magnetic field up to spherical harmonic degree 13 (above which the lithospheric field dominates so we cannot downward continue to the core), as well as of the radial SV and radial SA to degree 16 (the limit of what could be reliably estimated in CHAOS-6) at the core-mantle boundary in 2015 from the CHAOS-6 model are presented in Fig. 12.7. The field in 2015 is determined from vector and scalar field measurements, and also differences of along-track and acrosstrack field measurements made by $S w a r m$, as well as ground observatory vector field data (Finlay et al. 2016). Movies showing the time changes of such maps are available at www.spacecenter.dk/files/magnetic-models/CHAOS-6.

The radial field at the core-mantle boundary is characterized by high latitude flux concentrations over Canada and Siberia, and similarly in the Southern hemisphere under the edges of Antarctica towards South America and Australia. It is these features that give rise to the first-order dipolar structure of the geomagnetic field. Other striking features include a train of flux concentrations at low latitude under the Western hemisphere that have been observed to move westwards since the advent of continuous satellite observations in 1999, and the large concentration of reversed flux in the Southern hemisphere.

Turning to the time derivative of the field, known as the secular variation (SV), we find that regions of intense radial SV at the core surface occur close to edges of patches of strong radial field. Intense SV is observed in 2015 to lie in a broad band equatorward of $30^{\circ}$ latitude between longitudes $100^{\circ} \mathrm{E}$ and $90^{\circ} \mathrm{W}$ and is particularly associated with the westward movement of the intense low latitude flux patches. There is also a well-localized negative-positive-negative series of three patches of radial SV visible under Alaska and Siberia; this appears to be a consequence of the rapid westward movement of intense high latitude radial field concentrations. The $\mathrm{SV}$ is also generally high in the Asian longitudinal sector $60^{\circ}-120^{\circ} \mathrm{E}$.

The second time derivative of the radial field, or secular acceleration (SA) at the core-mantle boundary in 2015, displays a prominent positive-negative pair of foci under India-South East Asia, and a series of strong radial SA patches of alternating sign in the region under northern South America, as well as a positive-negative pair at high northern latitudes under Alaska-Siberia. In both the radial SV and SA, there is a striking absence of structure in the southern polar region (Holme et al. 2011; Olsen et al. 2014). Although the Pacific region shows weak radial SV (again see Holme et al. 2011; Olsen et al. 2014), in 2015, there was strong radial SA in the central Pacific, consistent with the aftermath of the jerk observed in 2014 at Hawaii. The SA also changes dramatically on sub-decadal time scales (Chulliat and Maus 2014; Chulliat et al. 2015; Finlay et al. 2015), exhibiting a series of pulses in amplitude. CHAOS-6 
Fig. 12.7 Radial component of the main field at the core-mantle boundary (radius $3480 \mathrm{~km}$ ) in 2015. Top (radial field up to degree 13) Middle (secular variation of radial field up to degree 16), Bottom (secular acceleration of the radial field up to degree 16). From the CHAOS-6 field model (Finlay et al. 2016)
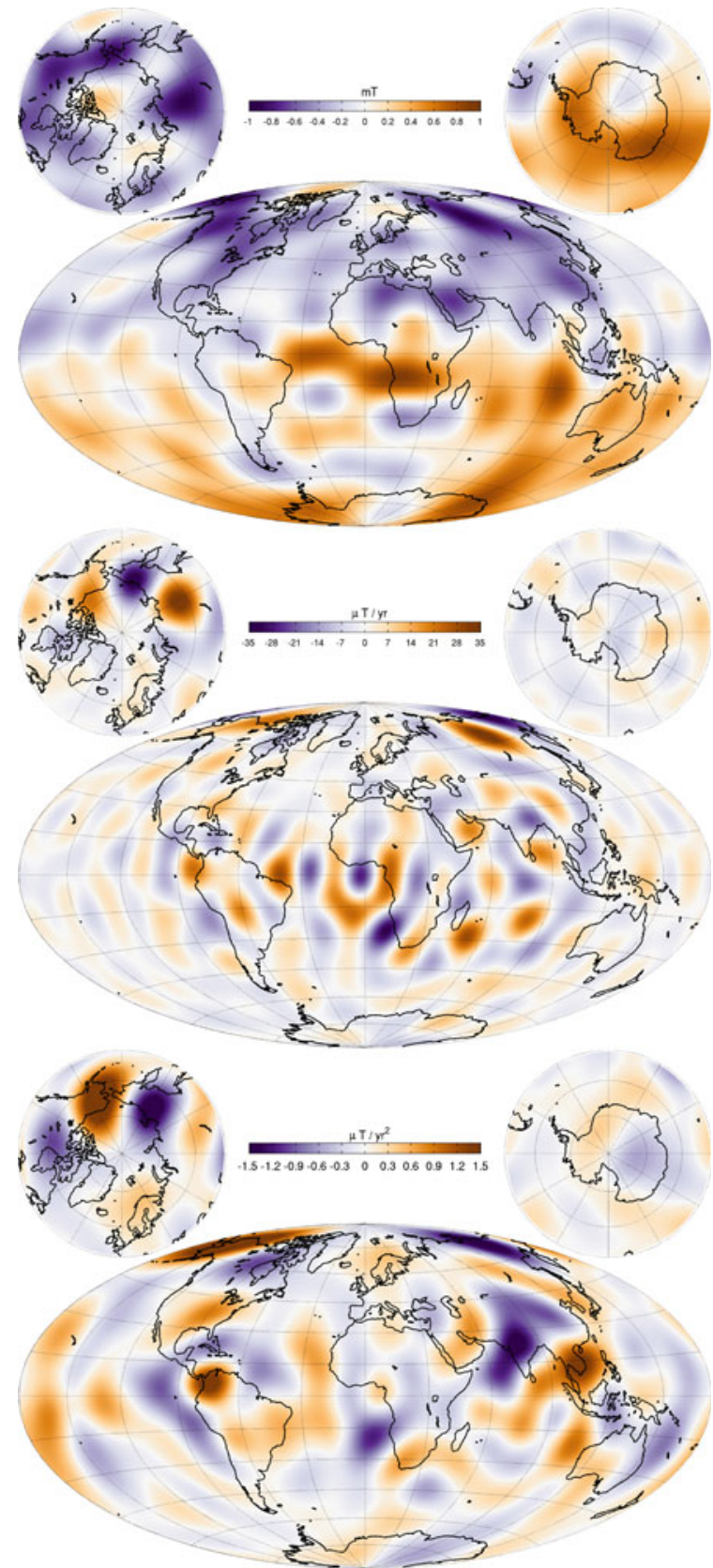
Fig. 12.8 As for Fig. 12.7, but with CHAOS-6 evaluated at satellite altitude of $400 \mathrm{~km}$
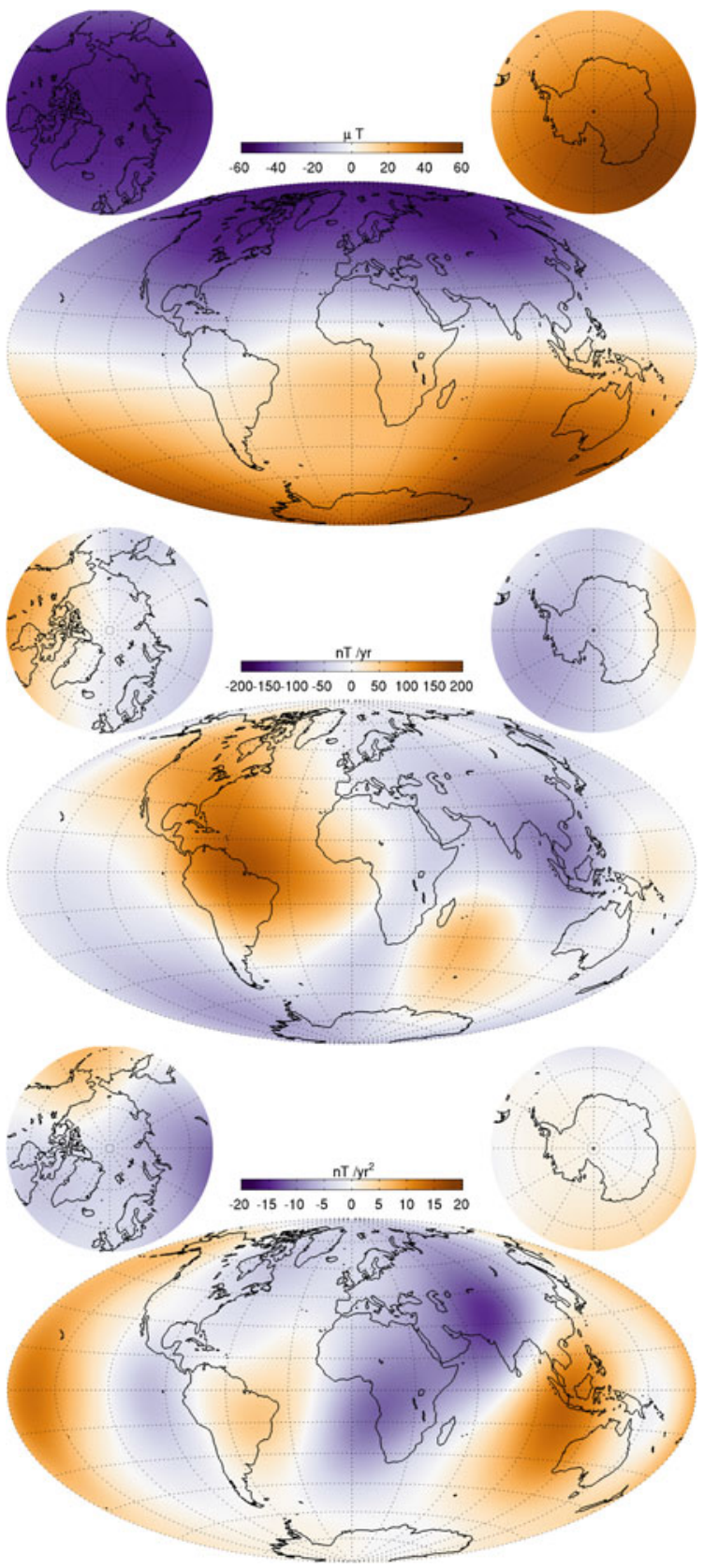
shows pulses of SA around 2006, 2009.5 and 2013. Maps and movies of the radial SA at the core surface also show recurring oscillations at particular locations, for example, under northern South America around $40^{\circ} \mathrm{W}$ close to the equator, and high amplitude SA is often found around longitude $100^{\circ} \mathrm{E}$.

Figure 12.8 presents the radial field, its SV and SA, from CHAOS-6, at a typical low-Earth-orbit satellite altitude of $400 \mathrm{~km}$. This is the internal field and its time changes that needs to be accurately accounted for when carrying out data reduction for ionospheric studies. The radial field at $400 \mathrm{~km}$ shows clear departures from a tilted dipole, with the high latitudes flux concentrations familiar from the core-mantle boundary again being evident. The radial field in the South Atlantic is distinctly weaker and there is a noticeable kink in the magnetic equator near South America. The radial field at satellite altitude is presently changing most rapidly at low latitudes in the American sector, while there are notable field accelerations taking place close to Indonesia and India-Pakistan, as well as in the mid-Pacific.

\subsubsection{The Lithospheric Field}

A recent map of the vertical field anomaly at the Earth's surface, due to the magnetized lithosphere is shown in Fig. 12.9 (top). This is derived from the LCS-1 model (Olsen et al. 2017) determined from CHAMP and Swarm field differences data and synthesized for spherical harmonic degrees $n=16-185$. The map shows the detailed structure of lithospheric field features throughout the world including the cratonic regions of the continents (especially Archean and Proterozoic domains) that show stronger anomalies, and the long wavelength features associated with and sub-parallel to the oceanic magnetic reversal stripes that are seen consistently on or near widely separated isochrones (green lines). In LCS-1, we see for the first time from the satellite data alone EW oceanic features associated with the reversal stripes formed during the last $50 \mathrm{Ma}$ of separation history of Australia from Antarctica. A number of other features on the ocean crust are evident. For example, there are NS trending lows in the vertical component map associated with the NS trending $85^{\circ} \mathrm{E}$ ridge in the Bay of Bengal.

The lithospheric power is higher in continental regions compared to oceanic regions, as expected due to the generally thicker continental/cratonic crust. The global average of $B_{r}^{2}$ at Earth's surface is $48.5 \mathrm{nT}^{2}$ (for spherical harmonic degrees $n=16-185)$, the power in continental regions is $66.1 \mathrm{nT}^{2}$ while that of the oceanic regions is only $39.4 \mathrm{nT}^{2}$, where the latter numbers are scaled to be whole Earth equivalent values. The LCS-1 model agrees well with the pre-Swarm satellite-data-based lithospheric field model MF7 (Maus 2010), up to its truncation degree of 133. If one wishes to study smaller scales of the lithospheric field, satellite data must be combined with near surface Aeromagnetic or marine survey data, for example as collected in the World Digital Magnetic Anomaly Map (WDMAM, currently in its second addition Lesur et al. 2016). Note, however, that the amplitude of small scale lithospheric field signals at satellite altitude is tiny. The bottom part of Fig. 12.9 
shows the vertical component of the lithospheric field at satellite altitude $(400 \mathrm{~km})$; the scale is then ten times smaller, and the lithospheric signal is on the order of a few to tens of nT, it is for this reason it must be accounted for when studying the magnetic signals due to ionospheric currents, especially when considering weaker current systems.

\subsection{Limitations of Present Main Field Models}

Although the present generation of main field models are rather impressive and extremely useful for studying ionospheric current systems, it is nonetheless important that users are aware of their limitations, and that space physicists realize that there is a clear opportunity for them to contribute in improving future main field models.

The major factor limiting the accuracy of the internal part of field models is the inability to correctly account for and remove all magnetospheric and ionospheric signals (Finlay et al. 2017). This includes the difficulty in modelling rapid changes in the magnetospheric field. Global coverage (requiring many days with the present satellite missions) are formally required to perform a separation into internal and external field components. Yet the magnetospheric field changes much faster on time scales of minutes to hours; present models try to account for this using activity indices based on ground-based observatory data but there are differences between ground and satellite data that remain poorly understood.

Another source of uncertainty is the internal signal due to currents induced in the electrically conducting mantle by the time-changing external field, which remains difficult to isolate. Present separations often rely on a priori models of the conductivity of the mantle and lithosphere, which although improving (e.g. Kuvshinov 2012) are subject to uncertainties.

Perhaps the most serious issue affecting the accuracy of today's internal field models come from the rapidly changing, high amplitude signals due to polar and auroral current systems that are driven by magnetosphere-ionosphere coupling. Most main field models seek to avoid the impact of strong field-aligned currents at satellite altitude by using only scalar intensity data (field aligned currents have magnetic perturbations that are small in the direction of the main field). However, even scalar data are affected by horizontal currents flowing in the polar ionospheric E-layer that result from the closure of field-aligned currents. These include eastward and westward auroral electrojet currents that can be large even in dark regions, particularly in association with substorm events. At present, no main field model is able to adequately represent these signals, which means (as they are internal to the satellites) their signature can be inadvertently be mapped in the estimated internal field. Attempts have been made to model the time-averaged signature of such horizontal polar ionospheric currents (e.g. Lesur et al. 2008; Olsen et al. 2016a). For example, the latter study included the following extra term in their internal potential model, describing signals arranged by quasi-dipole latitude $\theta_{Q D}$ and magnetic local time $\tau$ 

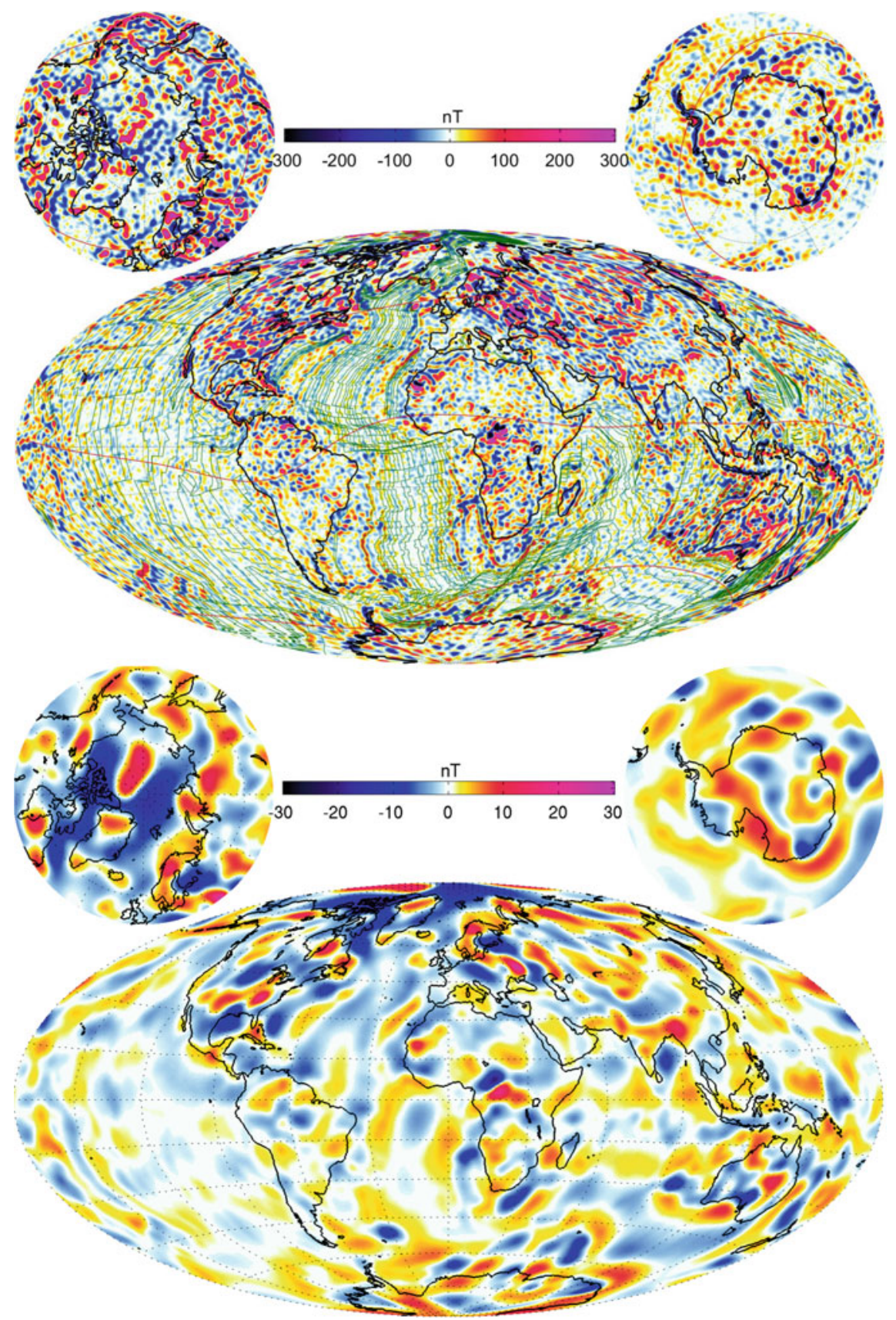

Fig. 12.9 Vertical field anomaly from the LCS-1 model of Olsen et al. (2017), synthesizing spherical harmonic degrees 16-185, at Earth's surface (top) and at a typical satellite altitude of $400 \mathrm{~km}$. Note the change in colour scale from a range of $\pm 300 \mathrm{nT}$ (surface, top) to $\pm 30 \mathrm{nT}$ ( $400 \mathrm{~km}$ altitude, bottom) 


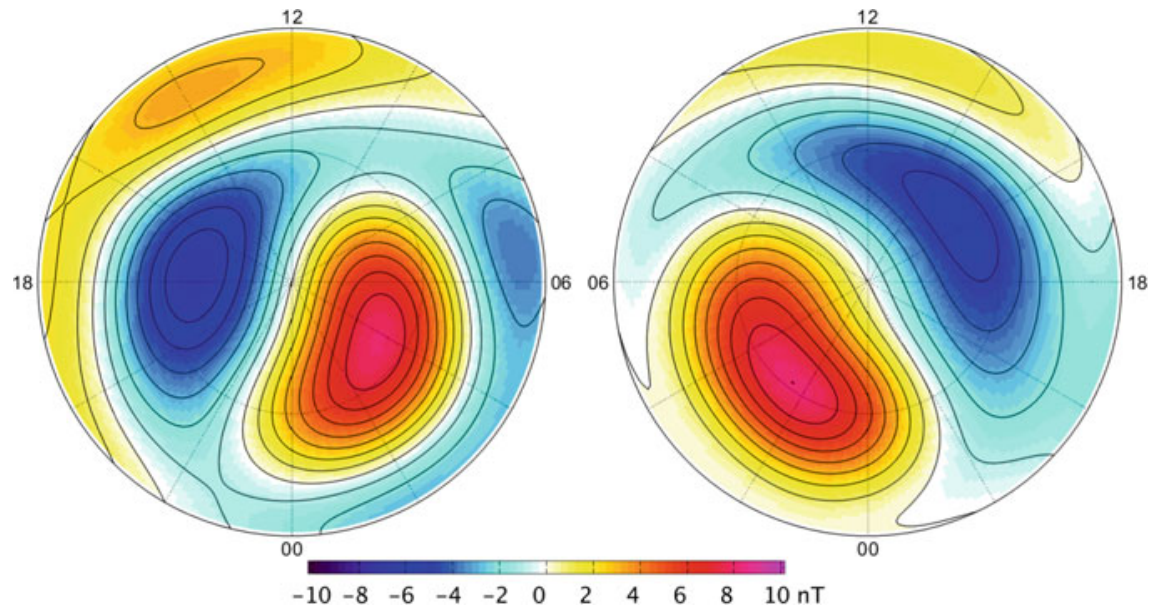

Fig. 12.10 Scalar magnetic field due to ionospheric currents, in dependence on QD latitude and magnetic local time (MLT, in hours) for the Northern (left) and Southern (right) polar regions as given by the potential $V_{M L T}$ of Eq. 12.20. The low-latitude boundary here is at \pm 60 quasi-dipole latitude. After Olsen et al. (2016a)

$$
V^{\mathrm{MLT}}=a \sum_{n=1}^{20} \sum_{m=1}^{n}\left(g_{n}^{m, \mathrm{MLT}} \cos m \tau+h_{n}^{m, \mathrm{MLT}} \sin m \tau\right)\left(\frac{a}{r}\right)^{n+1} P_{n}^{m}\left(\cos \theta_{Q D}\right)
$$

Figure 12.10 presents the resulting estimates of the time-averaged scalar magnetic field at satellite altitude from such sources, as a function of QD latitude and magnetic local time, derived from more than two years of Swarm data. There is much development still required of such models, in particular concerning how best to parameterize the time dependence of the signal, and how to more consistently account for the entire $3 \mathrm{D}$ current system in the polar region, including the influence of field-aligned currents on lower latitude vector field data (e.g. Laundal et al. 2016). This work urgently requires input from space physicists, especially those with expertise in studies of the polar ionosphere.

\subsection{Concluding Remarks}

Modern models of the main geomagnetic field are derived from multi-satellite data, and increasingly make use of along-track and inter-satellite field differences (i.e. approximate gradients) in order to reduce the signatures of large-scale magnetospheric sources and to enhance the signal of small-scale internal fields. Examples from the construction of two such models, CHAOS-6 and LCS-1 have been presented here in detail. Such advanced main field models, including contributions from the small-scale lithospheric field and accounting for the near-Earth signature of mag- 
netospheric sources, now enable more detailed study of relatively weak ionospheric current systems (Stolle et al. 2016).

Field gradient estimates can easily be incorporated within the conventional modelling framework as field differences, while also differencing the rows of the corresponding kernel matrices associated with each data point. It is very important to correctly specify the data uncertainty budget for vector, scalar and field gradient data, treating each satellite separately, in order for these to contribute appropriately during the field estimation procedure. Use of such procedures has enabled new details of the core field (in particular its time-dependent accelerations) and the lithospheric field (anomalies on scales down to $250 \mathrm{~km}$ ) to be imaged using data from the Swarm and CHAMP missions.

Internal field models are nevertheless certainly imperfect, in particular in the polar region. New ideas on how best to parameterize quiet-time field variations in the polar region are much needed; this represents a clear opportunity for the space physics community to apply their expertise in another domain.

Acknowledgements The author is grateful to Nils Olsen and Stavros Kotsiaros for collaboration on the work presented here, and for their help in preparing some of the material presented. Thanks also to Patrick Alken and Clemens Kloss for helpful comments on an early draft. The European Space Agency (ESA) is acknowledged for providing the Swarm data and for financially supporting the work on developing the Swarm Level 1 product 'Magnet'. The CHAMP mission was sponsored by the Space Agency of the German Aerospace Center (DLR) through funds of the Federal Ministry of Economics and Technology. The author thanks the International Space Science Institute in Bern, Switzerland, for supporting the ISSI Working Group: 'Multi-Satellite Analysis Tools, Ionosphere', from which this chapter resulted. The Editors thank Vincent Lesur for his assistance in evaluating this chapter.

\section{References}

Alken, P. 2016. Observations and modeling of the ionospheric gravity and diamagnetic current systems from champ and swarm measurements. Journal of Geophysical Research: Space Physics 121 (1): 589-601.

Backus, G.E. 1970. Non-uniqueness of the external geomagnetic field determined by surface intensity measurements. Journal of Geophysical Research 75 (31): 6339-6341.

Bloxham, J., and A. Jackson. 1992. Time-dependent mapping of the magnetic field at the coremantle boundary. Journal of Geophysical Research 97: 19. https://doi.org/10.1029/92JB01591.

de Boor, C. 1978. A practical guide to splines.

Chulliat, A., and S. Maus. 2014. Geomagnetic secular acceleration, jerks, and a localized standing wave at the core surface from 2000 to 2010. Journal of Geophysical Research: Solid Earth 119 (3): 1531-1543.

Chulliat, A., P. Alken, and S. Maus. 2015. Fast equatorial waves propagating at the top of the earth's core. Geophysical Research Letters 42 (9): 3321-3329.

Constable, C. 1988. Parameter estimation in non-gaussian noise. Geophysical Journal International 94 (1): 131-142.

Finlay, C.C., N. Olsen, and L. Tøffner-Clausen. 2015. Dtu candidate field models for igrf-12 and the chaos-5 geomagnetic field model. Earth, Planets and Space 67 (1): 114. 
Finlay, C.C., N. Olsen, S. Kotsiaros, N. Gillet, and L. Tøffner-Clausen. 2016. Recent geomagnetic secular variation from swarm. Earth, Planets and Space 68 (1): 1-18.

Finlay, C.C., V. Lesur, E. Thébault, F. Vervelidou, A. Morschhauser, and R. Shore. 2017. Challenges handling magnetospheric and ionospheric signals in internal geomagnetic field modelling. Space Science Reviews 206 (1-4):157. https://doi.org/10.1007/s11214-016-0285-9.

Friis-Christensen, E., H. Lühr, and G. Hulot. 2006. Swarm: A constellation to study the earth's magnetic field. Earth, Planets and Space 58 (4): 351-358.

Gubbins, D. 2004. Time series analysis and inverse theory for geophysicists. Cambridge University Press.

Herceg, M., P.S. Jørgensen, and J.L. Jørgensen. 2017. Characterization and compensation of thermoelastic instability of swarm optical bench on micro advanced stellar compass attitude observations. Acta Astronautica 137: 205-213.

Holme, R., N. Olsen, and F. Bairstow. 2011. Mapping geomagnetic secular variation at the coremantle boundary. Geophysical Journal International 186 (2): 521-528.

Jackson, A., and C. Finlay. 2007. Geomagnetic secular variation and its applications to the core. Treatise on Geophysics 5: 147-193.

Jørgensen, J., T. Denver, M. Betto, and P. Jorgensen. 2003. Microasc a miniature star tracker, small satellites for earth observations. In: Fourth International Symposium of the IAA, Berlin.

Jørgensen, J.L., E. Friis-Christensen, P. Brauer, F. Primdahl, P.S. Jørgensen, T.H. Allin, T. Denver et al. 2008. The swarm magnetometry package. In: Small Satellites for Earth Observation, 143 151. Springer.

Kauristie, K., A. Morschhauser, N. Olsen, C. Finlay, R. McPherron, J. Gjerloev, and H.J. Opgenoorth. 2017. On the usage of geomagnetic indices for data selection in internal field modelling. Space Science Reviews 206 (1-4): 61-90.

Kotsiaros, S. 2016. Toward more complete magnetic gradiometry with the swarm mission. Earth, Planets and Space 68 (1): 130.

Kotsiaros, S., C. Finlay, and N. Olsen. 2014. Use of along-track magnetic field differences in lithospheric field modelling. Geophysical Journal International 200 (2): 880-889.

Kuvshinov, A.V. 2012. Deep electromagnetic studies from land, sea, and space: Progress status in the past 10 years. Surveys in Geophysics 33(1):169-209. https://doi.org/10.1007/s10712-0119118-2.

Langel, R.A. 1987. The main field. Geomagnetism 1: 249-512.

Laundal, K.M., and A.D. Richmond. 2017. Magnetic coordinate systems. Space Science Reviews 206(1-4):27. https://doi.org/10.1007/s11214-016-0275-y.

Laundal, K.M., C.C. Finlay, and N. Olsen. 2016. Sunlight effects on the 3d polar current system determined from low earth orbit measurements. Earth, Planets and Space 68(1): 1. https://doi. org/10.1186/s40623-016-0518-x.

Lesur, V., I. Wardinski, M. Rother, and M. Mandea. 2008. Grimm: The GFZ reference internal magnetic model based on vector satellite and observatory data. Geophysical Journal International 173 (2): 382-394.

Lesur, V., I. Wardinski, M. Hamoudi, and M. Rother. 2010. The second generation of the GFZ reference internal magnetic model: GRIMM-2. Earth, Planets and Space 62 (10): 6.

Lesur, V., M. Rother, I. Wardinski, R. Schachtschneider, M. Hamoudi, and A. Chambodut. 2015a. Parent magnetic field models for the IGRF-12GFZ-candidates. Earth, Planets and Space 67 (1): $1-15$.

Lesur, V., K. Whaler, and I. Wardinski. 2015b. Are geomagnetic data consistent with stably stratified flow at the core-mantle boundary? Geophysical Journal International 201 (2): 929-946.

Lesur, V., M. Hamoudi, Y. Choi, J. Dyment, and E. Thébault. 2016. Building the second version of the world digital magnetic anomaly map (wdmam). Earth, Planets and Space 68 (1): 27.

Lowes, F. 1975. Vector errors in spherical harmonic analysis of scalar data. Geophysical Journal International 42 (2): 637-651. 
Lowes, F., and N. Olsen. 2004. A more realistic estimate of the variances and systematic errors in spherical harmonic geomagnetic field models. Geophysical Journal International 157 (3): 1027-1044.

Maus, S. 2010. Magnetic field model MF7.

Maus, S., and H. Lühr. 2005. Signature of the quiet-time magnetospheric magnetic field and its electromagnetic induction in the rotating earth. Geophysical Journal International 162 (3): 755 763.

Maus, S., H. Lühr, G. Balasis, M. Rother, and M. Mandea. 2005. Introducing pomme, the potsdam magnetic model of the earth. In: Earth Observation With Champ, Results From Three Years In Orbit, 293-298.

Maus, S., H. Lühr, and M. Purucker. 2006a. Simulation of the high-degree lithospheric field recovery for the swarm constellation of satellites. Earth, Planets and Space 58 (4): 397-407.

Maus, S., M. Rother, C. Stolle, W. Mai, S. Choi, H. Lühr, D. Cooke, and C. Roth. 2006b. Third generation of the potsdam magnetic model of the earth (pomme). Geochemistry, Geophysics, Geosystems 7 (7).

Maus, S., C. Manoj, J. Rauberg, I. Michaelis, and H. Lühr. 2010. Noaa/ngdc candidate models for the 11th generation international geomagnetic reference field and the concurrent release of the 6th generation pomme magnetic model. Earth, Planets and Space 62 (10): 2.

Olsen, N. 2002. A model of the geomagnetic field and its secular variation for epoch 2000 estimated from ørsted data. Geophysical Journal International 149 (2): 454-462.

Olsen, N., S. Kotsiaros. 2011. Geomagnetic observations and models. In: Magnetic Satellite Missions and Data IAGA, Special Sopron Book Series, vol. 5, 27-44. Dordrecht: Springer.

Olsen, N., and C. Stolle. 2017. Space Science Reviews 206: 5. https://doi.org/10.1007/s11214-016$0279-7$.

Olsen, N., L. Tøffner-Clausen, T.J. Sabaka, P. Brauer, J.M.G. Merayo, J.L. Jörgensen, J.M. Léger, O.V. Nielsen, F. Primdahl, and T. Risbo. 2003. Calibration of the ørsted vector magnetometer. Earth, Planets, and Space 55: 11-18.

Olsen, N., T.J. Sabaka, and F. Lowes. 2005. New parameterization of external and induced fields in geomagnetic field modeling, and a candidate model for IGRF 2005. Earth, Planets and Space 57 (12): 1141-1149.

Olsen, N., H. Lühr, T.J. Sabaka, M. Mandea, M. Rother, L. Tøffner-Clausen, and S. Choi. 2006. CHAOS-a model of the earth's magnetic field derived from CHAMP, ørsted, and SAC-C magnetic satellite data. Geophysical Journal International 166 (1): 67-75.

Olsen, N., M. Mandea, T.J. Sabaka, and L. Tøffner-Clausen. 2009. CHAOS-2-a geomagnetic field model derived from one decade of continuous satellite data. Geophysical Journal International 179 (3): 1477-1487.

Olsen, N., M. Mandea, T.J. Sabaka, and L. Tøffner-Clausen. 2010. The CHAOS-3 geomagnetic field model and candidates for the 11th generation IGRF. Earth, Planets and Space 62 (10): 1.

Olsen, N., H. Lühr, C.C. Finlay, T.J. Sabaka, I. Michaelis, J. Rauberg, and L. Tøffner-Clausen. 2014. The CHAOS-4 geomagnetic field model. Geophysical Journal International 197 (2): 815-827.

Olsen, N., G. Hulot, V. Lesur, C.C. Finlay, C. Beggan, A. Chulliat, T.J. Sabaka, R. Floberghagen, E. Friis-Christensen, R. Haagmans, et al. 2015. The swarm initial field model for the 2014 geomagnetic field. Geophysical Research Letters 42 (4): 1092-1098.

Olsen, N., C.C. Finlay, S. Kotsiaros, and L. Tøffner-Clausen. 2016a. A model of earth's magnetic field derived from 2 years of swarm satellite constellation data. Earth, Planets and Space 68 (1): 124.

Olsen, N., C. Stolle, R. Floberghagen, G. Hulot, and A. Kuvshinov. 2016b. Special issue "swarm science results after 2 years in space". Earth, Planets and Space 68 (1): 172.

Olsen, N., D. Ravat, C.C. Finlay, and L.K. Kother. 2017. LCS-1: A high resolution global model of the lithospheric magnetic field derived from champ and swarm satellite observations. Geophysical Journal International.

Riley, K.F., M.P. Hobson, and S.J. Bence. 2006. Mathematical methods for physics and engineering: a comprehensive guide. Cambridge University Press. 
Roberts, P.H., and E.M. King. 2013. On the genesis of the earth's magnetism. Reports on Progress in Physics 76(9):096801.

Sabaka, T.J., N. Olsen, and R.A. Langel. 2002. A comprehensive model of the quiet-time, near-earth magnetic field: phase 3. Geophysical Journal International 151 (1): 32-68.

Sabaka, T.J., N. Olsen, and M.E. Purucker. 2004. Extending comprehensive models of the earth's magnetic field with ørsted and CHAMP data. Geophysical Journal International 159 (2): $521-$ 547.

Sabaka, T.J., L. Tøffner-Clausen, and N. Olsen. 2013. Use of the comprehensive inversion method for swarm satellite data analysis. Earth, Planets and Space 65 (11): 2.

Sabaka, T.J., N. Olsen, R.H. Tyler, and A. Kuvshinov. 2015. CM5, a pre-swarm comprehensive geomagnetic field model derived from over $12 \mathrm{yr}$ of CHAMP, $\varnothing$ rsted, SAC-C and observatory data. Geophysical Journal International 200 (3): 1596-1626.

Sabaka, T.J., N. Olsen, R.H. Tyler, and A. Kuvshinov. 2018. A comprehensive model of earth's magnetic field determined from 4 years of swarm satellite observations. Earth Planets Space 70: 130. https://doi.org/10.1186/s40623-018-0896-3.

Seeber, G. 2003. Satellite geodesy: foundations, methods, and applications. Walter de gruyter

Stolle, C., I. Michaelis, and J. Rauberg. 2016. The role of high-resolution geomagnetic field models for investigating ionospheric currents at low earth orbit satellites. Earth, Planets and Space 68(1):1. https://doi.org/10.1186/s40623-016-0494-1.

Thébault, E., C. Finlay, P. Alken, C. Beggan, E. Canet, A. Chulliat, B. Langlais, V. Lesur, F. Lowes, C. Manoj, M. Rother, and R. Schachtschneider. 2015a. Evaluation of candidate geomagnetic field models for IGRF-12. Earth, Planets, and Space 67(112). https://doi.org/10.1186/s40623-0150273-4.

Thébault, E., C.C. Finlay, C.D. Beggan, P. Alken, J. Aubert, O. Barrois, F. Bertrand, T. Bondar, A. Boness, L. Brocco, et al. 2015b. International geomagnetic reference field: the 12th generation. Earth, Planets and Space 67 (1): 79.

Thébault, E., P. Vigneron, B. Langlais, and G. Hulot. 2016. A swarm lithospheric magnetic field model to SH degree 80. Earth, Planets and Space 68 (1): 126.

Tøffner-Clausen, L., V. Lesur, N. Olsen, and C.C. Finlay. 2016. In-flight scalar calibration and characterisation of the swarm magnetometry package. Earth, Planets, and Space 68: 129. https:// doi.org/10.1186/s40623-016-0501-6.

Winch, D., D. Ivers, J. Turner, and R. Stening. 2005. Geomagnetism and schmidt quasinormalization. Geophysical Journal International 160 (2): 487-504.

Yin, F., and H. Lühr. 2011. Recalibration of the CHAMP satellite magnetic field measurements. Measurement Science and Technology 22(5):055101.

Open Access This chapter is licensed under the terms of the Creative Commons Attribution 4.0 International License (http://creativecommons.org/licenses/by/4.0/), which permits use, sharing, adaptation, distribution and reproduction in any medium or format, as long as you give appropriate credit to the original author(s) and the source, provide a link to the Creative Commons license and indicate if changes were made.

The images or other third party material in this chapter are included in the chapter's Creative Commons license, unless indicated otherwise in a credit line to the material. If material is not included in the chapter's Creative Commons license and your intended use is not permitted by statutory regulation or exceeds the permitted use, you will need to obtain permission directly from the copyright holder. 